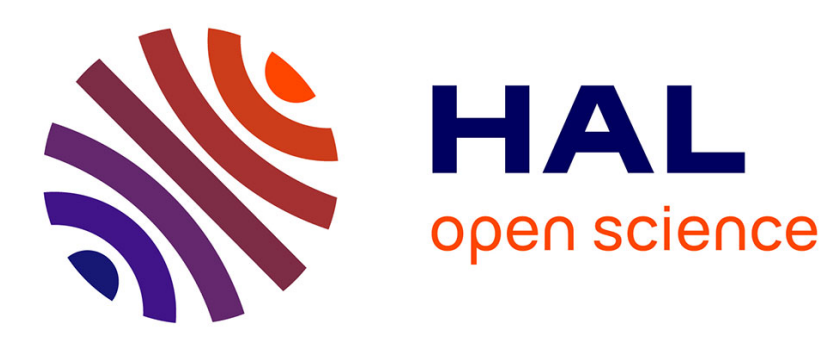

\title{
Modelling the evolution of complex conductivity during calcite precipitation on glass beads
}

Philippe Leroy, Shuai Li, Damien Jougnot, André Revil, Yuxin Wu

\section{To cite this version:}

Philippe Leroy, Shuai Li, Damien Jougnot, André Revil, Yuxin Wu. Modelling the evolution of complex conductivity during calcite precipitation on glass beads. Geophysical Journal International, 2017, 10.1093/gji/ggx001 . hal-01617337

\section{HAL Id: hal-01617337 \\ https://hal.science/hal-01617337}

Submitted on 26 Mar 2021

HAL is a multi-disciplinary open access archive for the deposit and dissemination of scientific research documents, whether they are published or not. The documents may come from teaching and research institutions in France or abroad, or from public or private research centers.
L'archive ouverte pluridisciplinaire HAL, est destinée au dépôt et à la diffusion de documents scientifiques de niveau recherche, publiés ou non, émanant des établissements d'enseignement et de recherche français ou étrangers, des laboratoires publics ou privés. 


\title{
Modelling the evolution of complex conductivity during calcite precipitation on glass beads
}

\author{
Philippe Leroy, ${ }^{1}$ Shuai Li, ${ }^{2}$ Damien Jougnot, ${ }^{3}$ André Revil ${ }^{4}$ and Yuxin $\mathrm{Wu}^{5}$ \\ ${ }^{1}$ BRGM, French geological survey, Orléans, France \\ ${ }^{2}$ Department of Earth Science and Engineering, Imperial College, London, United Kingdom. E-mail: lshuai8002@yahoo.com \\ ${ }^{3}$ Sorbonne Universités, UPMC Univ. Paris 06, CNRS, EPHE, UMR 7619 METIS, Paris, France \\ ${ }^{4}$ ISTerre, CNRS, UMR CNRS 5275, Université Savoie Mont-Blanc, Le Bourget du Lac, France \\ ${ }^{5}$ Climate and Ecosystem Sciences Division, Lawrence Berkeley National Laboratory, Berkeley, CA, USA
}

Accepted 2017 January 4. Received 2016 December 31; in original form 2016 May 31

\begin{abstract}
SUMMAR Y
When $\mathrm{pH}$ and alkalinity increase, calcite frequently precipitates and hence modifies the petrophysical properties of porous media. The complex conductivity method can be used to directly monitor calcite precipitation in porous media because it is sensitive to the evolution of the mineralogy, pore structure and its connectivity. We have developed a mechanistic grain polarization model considering the electrochemical polarization of the Stern and diffuse layers surrounding calcite particles. Our complex conductivity model depends on the surface charge density of the Stern layer and on the electrical potential at the onset of the diffuse layer, which are computed using a basic Stern model of the calcite/water interface. The complex conductivity measurements of Wu et al. on a column packed with glass beads where calcite precipitation occurs are reproduced by our surface complexation and complex conductivity models. The evolution of the size and shape of calcite particles during the calcite precipitation experiment is estimated by our complex conductivity model. At the early stage of the calcite precipitation experiment, modelled particles sizes increase and calcite particles flatten with time because calcite crystals nucleate at the surface of glass beads and grow into larger calcite grains. At the later stage of the calcite precipitation experiment, modelled sizes and cementation exponents of calcite particles decrease with time because large calcite grains aggregate over multiple glass beads and only small calcite crystals polarize.
\end{abstract}

Key words: Electrical properties; Hydrogeophysics; Microstructure; Permeability and porosity.

\section{INTRODUCTION}

Calcite is one of the most abundant minerals in the Earth's crust and frequently precipitates when $\mathrm{pH}$ and alkalinity increase (Vancappellen et al. 1993; Stipp 1999). Calcite precipitation modifies the rock porosity, and can have either benefits or side effects for the mechanical and transport properties of porous media. Calcite precipitation in porous media has broad applications in geotechnical engineering for soil strengthening (DeJong et al. 2006, 2010; Whiffin et al. 2007) and in environmental studies for the sequestration of heavy metals (Sturchio et al. 1997; Cheng et al. 1999), radionuclides (Fujita et al. 2004; Mitchell \& Ferris 2005), and $\mathrm{CO}_{2}$ in geological formations (Pruess et al. 2003; Xu et al. 2003). However, calcite precipitation can also have undesirable effects such as the decrease of the permeability of reactive barriers for the remediation of aquifers (Wilkin et al. 2003; Scheibe et al. 2006; Jeen et al. 2007).

Calcite precipitation in porous media can be observed in situ by conventional monitoring approaches using geochemical analyses of borehole samples (Scheibe et al. 2006; Li et al. 2009, 2011). Geochemical borehole data give accurate measurements of the water chemical composition associated with calcite precipitation but they are time consuming, not continuous in time, invasive and spatially limited to the location of the boreholes. The complex conductivity method can be used to non-intrusively monitor calcite precipitation in porous media because of its sensitivity to the evolution of the mineralogy, pore structure and connectivity (Wu et al. 2010, 2011; Zhang et al. 2012). This geoelectrical method investigates both conduction (i.e. electromigration of the charge carriers) and polarization (i.e. the reversible storage of electrical charges) of porous materials (Kemna et al. 2012). Polarization of oxides and carbonates minerals such as calcite is related to the polarization of the electrical double layer (EDL) surrounding the particles under an external alternating current or electrical field (Chelidze \& Gueguen 1999; Jougnot et al. 2010; Revil \& Florsch 2010). When a sinusoidal current at a range of frequencies is applied to the charged porous medium and the resulting difference of electrical potential is measured, complex conductivity is referred to as spectral induced 
polarization (SIP) (Collet 1990; Weller \& Borner 1996; Luo \& Zhang 1998; Huisman et al. 2015).

At low frequencies (typically $<1 \mathrm{kHz}$ ), there are two major phenomena controlling electrical charge transport in porous media containing no metallic particles: the complex conductivity of the bulk pore water associated with the electromigration of charge carriers in the interconnected, liquid-filled pore space and the complex interfacial or surface conductivity associated with the conduction and polarization processes in the EDL (Dukhin \& Shilov 1974; Vinegar \& Waxman 1984; Delima \& Sharma 1992; Lesmes \& Morgan 2001; Leroy et al. 2008; Revil 2012). Conduction in bulk water strongly depends on the water chemical composition, the pore volume occupied by the liquid and the topology of the pore space (i.e. related to transport properties, Revil et al. 2005; Jougnot et al. 2009). While the real part of complex conductivity is sensitive to electromigration processes in the pore space and at the mineral surface, its imaginary part is sensitive to polarization processes at the mineral surface (Weller et al. 2013; Okay et al. 2014). The magnitude of the imaginary conductivity is controlled by the electrochemical properties and the surface area of the mineral/water interface (Vaudelet et al. 2011; Bucker \& Hordt 2013; Okay et al. 2014). The frequency behaviour of the imaginary conductivity is controlled by relaxation of ions in the EDL, which depends on the size, shape, surface roughness of the particles or pores, and on the ionic surface mobility at the mineral/water interface (Leroy et al. 2008; Bucker \& Hordt 2013).

Wu et al. (2010) performed complex conductivity measurements and modelling of calcite precipitation on a column packed with glass beads. The evolution of calcite precipitation in porous media was clearly observed from their imaginary conductivity data. The empirical Cole-Cole model (Cole \& Cole 1941; Pelton et al. 1978) was used by Wu et al. (2010) to interpret the complex conductivity signature of calcite precipitation on glass beads in terms of mean relaxation time and chargeability (capacity of the porous medium to store electric charges). At the early stage of the calcite precipitation experiment, $\mathrm{Wu}$ et al. (2010) observed that the mean relaxation time and chargeability increase with time, and, after nine days of the experiment, they observed that the mean relaxation time and chargeability decrease with time. Wu et al. (2010) considered that discrete micrometric calcite particles polarize and grow during the early stage of calcite precipitation and that large millimetric calcite aggregates do not polarize sufficiently over multiple glass beads, that is only remaining and new discrete calcite particles polarize after nine days of experiment. However, the lack of physical processes in the Cole-Cole model to interpret the complex conductivity data restricts the understanding of the effects of calcite precipitation on the evolution of the mineralogy, pore structure and connectivity in the glass beads column. For instance, the Cole-Cole model gives no information on the evolution of the size and shape of calcite particles during their precipitation. Furthermore, the Cole-Cole model gives no information about the phenomena at the mineral/water interface responsible for the SIP response, such as the surface concentration and diffusivity of the ions in the EDL.

Leroy et al. (2008) proposed a grain polarization model accounting for the surface site density of adsorbed counter-ions in the Stern layer at the mineral/water interface to describe the complex conductivity response of glass beads at different salinities. Their complex conductivity model considers the electrochemical polarization of the Stern layer following Schwarz theory (Schwarz 1962) and was combined with a triple layer model (TLM) of the silica/water interface. The differential effective medium theory (DEM; Bruggeman 1935; Hanai 1968; Sen et al. 1981) was used by Leroy et al. (2008)

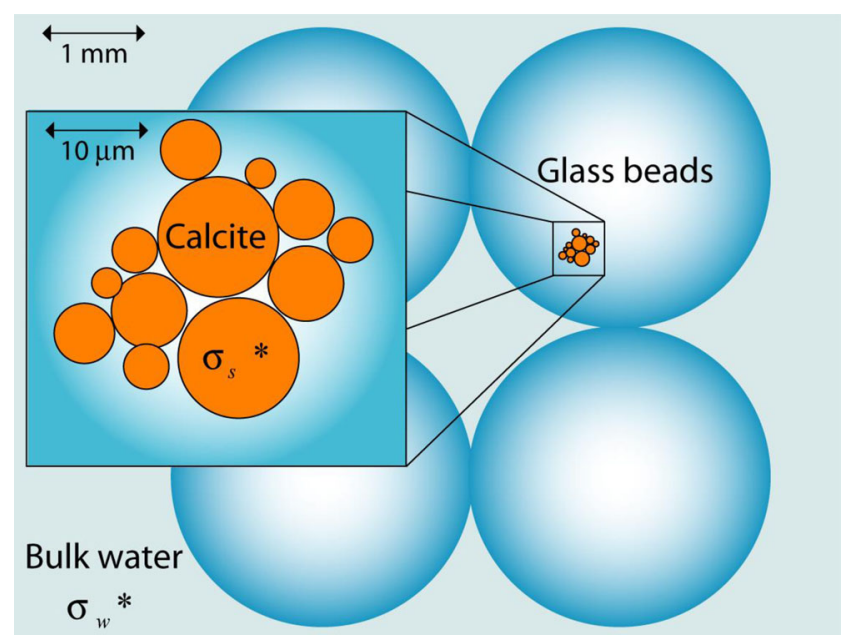

Figure 1. Sketch of our complex conductivity model of the porous medium. Parameters $\sigma_{w}^{*}$ and $\sigma_{s}^{*}$ are the complex bulk water and surface conductivity of solid, respectively. Calcite particles are mostly rhombohedral, but considering them spherical is a good first approximation.

to compute the complex conductivity of the porous sample considering bulk water complex conductivity, complex surface conductivity of the glass beads, porosity and grain shape. Their complex conductivity model successfully reproduced the measured phase shift (between injected current and measured electrical potential difference) of glass beads as function of frequency. Leroy et al. (2008) showed that two distinct peaks in the imaginary conductivity successfully capture the relaxation times associated with grain size distribution and surface roughness. However, the grain polarization model of Leroy et al. (2008) was restricted to monovalent electrolytes and did not consider the effects of the diffuse layer on the Stern layer polarization.

The induced polarization of calcite precipitates needs to be further clarified using a mechanistic complex conductivity model accounting for the EDL properties, the particle size distribution and shape. In this study, a mechanistic model for the induced polarization of calcite is proposed, which depends on the surface charge density and ion mobility in the Stern layer, electrical potential at the onset of the diffuse layer, particle size distribution and shape. Our SIP model is combined with a basic Stern model (BSM) of the calcite/water interface (Li et al. 2016). After a short review of the grain polarization model of Leroy et al. (2008), the complex conductivity model is generalized to the polarization of different monovalent and multivalent counter-ions in the Stern and diffuse layers according to Lyklema et al. (1983). Then, the predictions of the model are compared to the imaginary conductivity data of $\mathrm{Wu}$ et al. (2010), and the evolution of the particles sizes and shapes during calcite precipitation on glass beads is estimated accordingly.

\section{THEORETICAL BACKGROUND}

\subsection{Complex conductivity model of the porous medium}

We consider a porous medium containing particles, that is glass beads (of millimetric size) and calcite crystals (of micrometric size), and pore water. A sketch of our complex conductivity model is presented in Fig. 1. The differential effective medium theory (Bruggeman 1935; Hanai 1968; Sen et al. 1981) is used to compute the complex conductivity of the porous medium according to the complex conductivity of the particles and water. In the DEM theory, 
solid particles are embedded in a self-similar way in the pore water. First, a particle is added to the liquid and the influence of the particle upon the conductivity of the medium is calculated. Then, the particle/liquid mixture is used to coat a second particle and the influence of the second particle upon the conductivity of the medium is calculated, and so on following an iterative procedure until the desired porosity is reached. The DEM theory assumes that the pore space of granular media is interconnected. It works well to describe the electrical properties of uncompacted and uncemented granular media with small contiguity between the grains (Revil 2000). The complex conductivity of the porous medium $\sigma^{*}$ is calculated using the following equations (Revil \& Cathles 1999):

$\sigma^{*}=\frac{\sigma_{w}^{*}}{F}\left(\frac{1-\sigma_{s}^{*} / \sigma_{w}^{*}}{1-\sigma_{s}^{*} / \sigma^{*}}\right)^{m}$,

$F=\phi^{-m}$,

where $\sigma_{w}^{*}$ is the complex conductivity of the bulk water (in $\mathrm{S} \mathrm{m}^{-1}$ ), $\sigma_{s}^{*}$ is the complex surface conductivity and $m$ is the cementation exponent of the particles ( $m=1.5$ for spheres, Sen et al. 1981). The parameter $F$ is the electrical formation factor of the porous medium and $\phi$ is the interconnected porosity corresponding to the volume of the connected pores divided by the total volume of the porous medium.

The complex conductivity of the bulk water $\sigma_{w}^{*}$ is calculated considering conduction and dielectric displacement currents and is given by (Schwarz 1962):

$\sigma_{w}^{*}=\sigma_{w}+i \omega \varepsilon_{w}$,

where $\sigma_{w}$ is the DC (direct current) bulk water conductivity, $\omega$ is the angular frequency of the applied sinusoidal current (in $\operatorname{rad~s}^{-1}, \omega=$ $2 \pi f$ with $f$ being the frequency in hertz or $\left.\mathrm{s}^{-1}\right), i$ is the imaginary number $\left(i^{2}=-1\right)$, and $\varepsilon_{w}$ is the dielectric permittivity of water (in $\mathrm{F} \mathrm{m}^{-1}$ ). Water dielectric permittivity is written as $\varepsilon_{w}=\varepsilon_{r} \varepsilon_{0}$, where $\varepsilon_{r}$ is the relative dielectric permittivity of water $\left(\varepsilon_{r} \cong 78.3\right.$ for bulk water at a pressure of 1 bar and a temperature $T$ of $298 \mathrm{~K})$ and $\varepsilon_{0}$ is the dielectric permittivity of vacuum $\left(\varepsilon_{0} \cong 8.854 \times 10^{-12} \mathrm{~F} \mathrm{~m}^{-1}\right)$ (Lide 1990).

The complex surface conductivity of the particles of different sizes $\sigma_{s}^{*}$ is calculated considering the superposition principle (Lesmes \& Morgan 2001). It is computed using a volumetric mixing formula assuming that the electrochemical conductions and polarizations of particles of different sizes all add in parallel, that is that the complex conductivity response of particles of same size is weighted by the relative volume of the solid that is occupied by these particles. The resulting complex surface conductivity of the particles depends on the particle size distribution (PSD) $f\left(d_{i}\right)$, with $d_{i}$ the particle diameter (in $\mathrm{m}$ ), and is given by (Lesmes \& Morgan 2001; Leroy et al. 2008):

$\sigma_{s}^{*}=\sum_{i=1}^{Q} f\left(d_{i}\right) \sigma_{s}^{*}\left(d_{i}, \omega\right)$,

where $Q$ is the number of different diameters. The normalization of the PSD implies:

$\sum_{i=1}^{Q} f\left(d_{i}\right)=1$,

which means that $f\left(d_{i}\right)$ is in fact a weight coefficient depending entirely on the PSD.

In the following section, the complex surface conductivity of one particle, $\sigma_{s}^{*}\left(d_{i}, \omega\right)$, is described using a mechanistic model of con- duction and induced polarization. The model is mechanistic because it is based on the electrochemical properties of the EDL around particles. This complex conductivity model considers ion electromigration (conduction) in the diffuse layer, which is assumed to form a dielectric medium around particles, and ion electromigration and back-diffusion, that is polarization of the discontinuous Stern layers around particles. Polarization of the Stern layer is compensated by diffuse layer polarization to ensure electroneutrality of the particle in water.

\subsection{Complex conductivity model of the particle}

\subsubsection{Surface conductivity models of Lyklema et al. (1983) and Leroy et al. (2008)}

The complex surface conductivity of the particle is computed using the complex conductivity model of Leroy et al. (2008) generalized to the polarization of the Stern and diffuse layer containing monovalent and multivalent ions using the approach of Lyklema et al. (1983) (Appendices A and B). The complex surface conductivity model of Leroy et al. (2008) is based on the Schurr theory (Schurr 1964), which combines Schwarz theory (Schwarz 1962) for the AC (alternating current) conductivity (polarization) and O'Konski theory (O'Konski 1960) for the DC conductivity (electromigration). Following Schwarz theory, Leroy et al. (2008) considered that the surface density of one type of monovalent counter-ion in the Stern layer is responsible for particle polarization, whereas Lyklema et al. (1983) considered directly the effects of the total surface charge density of the Stern and diffuse layer on particle polarization, hence extending Schwarz theory to the polarization of the Stern and diffuse layer containing monovalent and multivalent ions.

Leroy et al. (2008) therefore assumed that movement of ions in the Stern layer around particles controls the observed phase shift between injected sinusoidal current and measured electrical potential difference when SIP experiments are performed on porous media containing no metallic particles. The Stern layer is a compact layer of counter-ions at the mineral/water interface compensating the surface charge of the mineral (Stern 1924; Leroy \& Revil 2004). Leroy et al. (2008) considered the electrochemical polarization of the discontinuous Stern layers around the particles, that is in their model counter-ions in the Stern layer were assumed to electromigrate along the particle surface (under the applied alternating electrical field) and to diffuse back in the opposite direction to re-establish their initial homogenous distribution as long as the frequency is low (Fig. 2). Therefore, a relaxation time or characteristic frequency (inverse of the relaxation time) can be associated with the shape and size of the particle and with the tangential diffusion coefficient of the counterions in the Stern layer. Typically, large micrometric particles or small nanometric particles with a high degree of surface roughness induce high relaxation times and small characteristic frequencies (typically < kHz) (Chelidze \& Gueguen 1999; Lesmes \& Morgan 2001). These ion movements in the Stern layer were also assumed to control not only the frequency behaviour but also the magnitude of the polarization process, that is high surface density of mobile charges in the Stern layer induces high particle polarization.

The diffuse layer is made of counter-ions and co-ions, is located further away from the mineral surface compared to the Stern layer, and electrostatically compensates the surface charge of the mineral not compensated by the Stern layer (Gouy 1910; Chapman 1913). The diffuse layer is not as compacted as the Stern layer, generally thicker than the Stern layer, and its thickness decreases significantly 


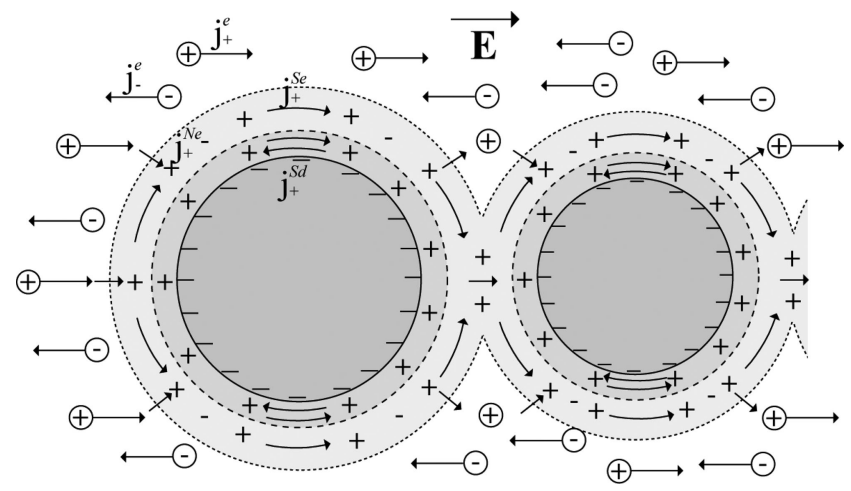

Figure 2. Sketch of the complex conductivity model of Leroy et al. (2008) for particles of different sizes surrounded by discontinuous Stern layers and overlapping diffuse layers in a saline aqueous solution. Only the discontinuous Stern layer is assumed to polarize around particles. The bulk water and continuous diffuse layer only contribute to electromigration fluxes $\mathbf{j}_{ \pm}^{e}$ and hence do not polarize. Bulk water and diffuse layer exchange ions via normal ions fluxes $\mathbf{j}_{ \pm}^{N e}$ and tangential ions fluxes $\mathbf{j}_{ \pm}^{S e}$ occur in the diffuse layer. Tangential electromigration and counter-balancing diffusion fluxes, $\mathbf{j}_{+}^{S e}$ and $\mathbf{j}_{+}^{S d}$, respectively, occur in the Stern layer. No exchange of ions is considered between the Stern and diffuse layers (modified, from Okay et al. 2014).
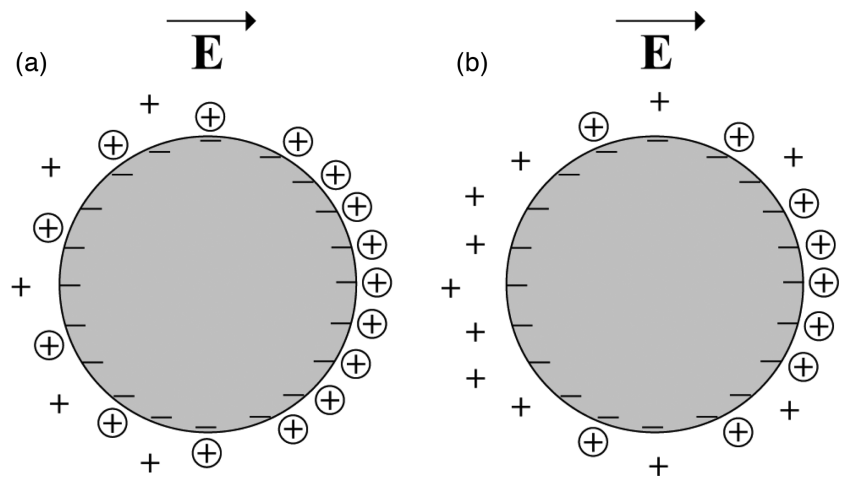

Figure 3. Sketch of the polarized Stern and diffuse layers in the case of a weak diffuse layer (a) and a strong diffuse layer (b) (modified, from Lyklema et al. 1983). Counter-ions in the Stern layer electromigrate and diffuse back over a shorter distance when diffuse layer becomes stronger.

when ionic strength of surrounding water increases (Hunter 1981). Leroy et al. (2008) considered that the diffuse layer is continuous at the scale of the porous medium and hence does not polarize because particles are in contact with each other (Leroy \& Revil 2009; Okay et al. 2014; Fig. 2). Leroy et al. (2008) also assumed that only electromigration currents in the diffuse layer and bulk water control the DC conductivity of the porous medium. However, according to Lyklema et al. (1983), the Stern layer is almost completely screened by the diffuse layer and hence the polarization of the diffuse layer cannot be ignored. The diffuse layer has to polarize to compensate the polarized Stern layer in order to ensure electroneutrality of the solid/water interface. Lyklema et al. (1983) considered the effects of the tangential oscillations of the diffuse layer on the polarization of bound counter-ions in the Stern layer. These authors considered that the polarized diffuse layer decreases the relaxation time of the polarized Stern layer (Fig. 3). Grosse \& Foster (1987) considered ion diffusion effects in the bulk electrolyte surrounding the polarized counter-ions layer and also emphasized that ion diffusion outside the Stern layer decreases the relaxation time of bound counter-ions in the Stern layer.

\subsubsection{The new surface conductivity model}

The complex surface conductivity of a charged spherical particle of diameter $d$ immersed in an aqueous electrolyte is calculated considering conduction and dielectric displacement currents and is given by (O'Konski 1960):

$\sigma_{s}^{*}=\sigma_{s}+i \omega \varepsilon_{s}$,

$\sigma_{s}=\frac{4}{d} \Sigma_{s}$

$\varepsilon_{s}=\alpha \rho_{s} \varepsilon_{0}$,

where $\sigma_{s}$ is the complex surface conductivity of the particle calculated according to Ohm's law (excluding dielectric displacement currents), $\varepsilon_{s}$ is the dielectric permittivity, $\Sigma_{s}$ is the specific surface conductivity (in $\mathrm{S}$ ), and $\rho_{s}$ is the volumetric density (in $\mathrm{kg} \mathrm{m}^{-3}$ ) of the particles. Eq. (8) is an empirical equation linking the dielectric permittivity of the mineral to its volumetric density and is taken from the study of Olhoeft (1981) $\left(\alpha=0.00191 \mathrm{~m}^{3} \mathrm{~kg}^{-1}\right.$ in the absence of lossy materials, such as magnetic or metallic minerals, and Fe Ti sulfides oxides such as ilmenite). It should be noted that calcite particles are assumed to be spherical in eq. (7), but the shape of calcite crystals is in reality mostly rhombohedral (Kile et al. 2000). Nevertheless, we assume that the spherical particles assumption for the complex surface conductivity of calcite particles is a fairly good first-order approximation.

The specific surface conductivity of the particle is calculated considering the specific surface conductivity of the Stern layer (a complex quantity) added to the DC specific surface conductivity (a real quantity) (superposition principle), and is described using the following equations (Appendices $\mathrm{A}$ and $\mathrm{B}$ ):

$$
\begin{aligned}
& \Sigma_{s}=\Sigma_{s}^{b}\left(\frac{i \omega \tau_{b}}{1+i \omega \tau_{b}}\right)+\Sigma_{s}^{\mathrm{DC}}, \\
& \tau_{b}=\frac{1}{2 \pi f_{b}}=\frac{a^{2}}{2 D_{b} M}=\frac{d^{2}}{8 D_{b} M}=\frac{d^{2}|q|}{8 k_{B} T \beta_{b} M},
\end{aligned}
$$

where $\Sigma_{s}^{b}$ and $\Sigma_{s}^{D C}$ are, respectively, the contribution of the Stern layer (subscript ' $b$ ' for the beta-plane corresponding to the Stern plane) and the DC contribution of the EDL to the specific surface conductivity of the particle, $M$ is a parameter (dimensionless) characterizing the effects of the diffuse layer polarization on the Stern layer polarization $(M \geq 1)$ (Lyklema et al. 1983; Niu et al. 2016), and $\tau_{b}$ is the mean relaxation time (in s) of the counter-ions in the Stern layer. The parameter $f_{b}$ is the critical or characteristic frequency (in $\mathrm{Hz}$ ) corresponding to the electrochemical polarization of the Stern layer, $D_{b}$ is the surface diffusion coefficient of the counter-ions in the Stern layer (in $\mathrm{m}^{2} \mathrm{~s}^{-1}$ ) and $a$ is the radius of the particle (in $\mathrm{m}$ ). The surface diffusion coefficient of the counterions in the Stern layer is expressed as a function of the surface mobility of the counter-ions in the Stern layer $\beta_{b}\left(\right.$ in $\left.\mathrm{m}^{2} \mathrm{~s}^{-1} \mathrm{~V}^{-1}\right)$ using the Nernst-Einstein equation $D_{b}=k_{B} T \beta_{b} /|q|$ where $k_{B}$ is the Boltzmann constant (of value $\sim 1.381 \times 10^{-23} \mathrm{~J} \mathrm{~K}^{-1}$ ), $T$ is the temperature (in $\mathrm{K}$ ), and $q$ is the ionic charge in the Stern layer (in C).

We consider that only the diffuse layer contributes to the DC surface conductivity of calcite particles because, following Schwarz (1962), all ions in the polarized Stern layer are assumed to diffuse back to recover their initial distribution (at thermodynamic equilibrium) and to not exchange with ions in the diffuse layer and bulk aqueous electrolyte. In the following text, we will therefore consider that $\Sigma_{s}^{d}=\Sigma_{s}^{D C}$ with $\Sigma_{s}^{d}$ the DC contribution of the diffuse layer 
to the specific surface conductivity. Even if the diffuse layer compensates the Stern layer polarization and necessarily polarizes, we do not consider a diffuse layer polarization mechanism as defined for instance in Dukhin \& Shilov (1974) or in Fixman (Pacios et al. 2016) theories involving volume polarization effects inside and outside the diffuse layer (Delgado et al. 1998). Therefore, we assume that the Stern layer contributes to surface polarization currents and the diffuse layer to surface conduction currents. Therefore, in our new complex conductivity model, only the Stern layer contributes to the imaginary part of the complex conductivity, the quadrature conductivity of the sample. The diffuse layer decreases the relaxation time of the polarized Stern layer and contributes to the real part of the complex conductivity, the in-phase conductivity of the sample.

Following Lyklema et al. (1983) for the polarization of the Stern layer (see Appendices A and B) and Revil \& Glover (1997) for ions electromigration in the diffuse layer, the contributions of the Stern and diffuse layers to the specific surface conductivity of calcite are written as:

$\Sigma_{s}^{b}= \pm \beta_{b} Q_{b}$

$\Sigma_{s}^{d}=\sum_{i=1}^{N} e z_{i} B_{i}^{d} \Gamma_{i}^{d}$

where

$B_{i}^{d}=\beta_{i}^{d}+\frac{2 \varepsilon_{w} k_{B} T}{\eta_{w} e z_{i}} \cong \beta_{i}^{w}+\frac{2 \varepsilon_{w} k_{B} T}{\eta_{w} e z_{i}}$

denotes an effective ion mobility in the diffuse layer (in $\mathrm{m}^{2} \mathrm{~s}^{-1} \mathrm{~V}^{-1}$ ), and where ' + ' stands for cations and ' - ' stands for anions in the Stern layer, $Q_{b}$ is the surface charge density of the Stern layer (in $\left.\mathrm{C} \mathrm{m}^{-2}\right), N$ is the number of different ions in the diffuse layer, $e$ is the elementary charge (of value $\sim 1.602 \times 10^{-19} \mathrm{C}$ ), $z_{i}$ is the ion valence, and $\Gamma_{i}^{d}$ is the surface site density of ions $i$ in the diffuse layer (in sites $\mathrm{m}^{-2}$ ). The parameter $\beta_{i}^{d}$ is the ion mobility in the diffuse layer due to electromigration (in $\mathrm{m}^{2} \mathrm{~s}^{-1} \mathrm{~V}^{-1}$ ) and $\eta_{w}$ is the dynamic viscosity of bulk water (in Pa s), $\eta_{w} \cong 0.8903 \times 10^{-3} \mathrm{~Pa}$ s for pure water at $T=298 \mathrm{~K}$ (Lide 1990). The ions effective mobility in the diffuse layer, $B_{i}^{d}$, considers electromigration (first term of eq. 13) and electro-osmosis (second term of eq. 13) (Bikerman 1940; Revil \& Glover 1997; Heuser et al. 2012; Leroy et al. 2015). Because the diffuse layer is far (several $\AA$ ) from the mineral surface and contains mostly counter-ions slowing down slightly counter-ions electromigration compared to co-ions (Bernard et al. 1992), we assume that the ion mobility in the diffuse layer is similar to the ion mobility in the bulk water, that is $\beta_{i}^{d} \approx \beta_{i}^{w}$ (Lyklema \& Minor 1998; Leroy et al. 2008; Leroy \& Revil 2009).

It should be noted that the original Schwarz complex conductivity model (Schwarz 1962) considers a monovalent counter-ion of charge $e$ at the solid/water interface. We follow the approach of Lyklema et al. (1983) to extend the Schwarz (1962) polarization model to different counter-ions adsorbed in the Stern layer (represented by the surface charge density $Q_{b}$ ) by assuming that the different counter-ions in the Stern layer have the same tangential mobility (Appendix A). The tangential ionic mobility in the Stern layer has never been measured and hence remains unknown and dependent on the model to interpret electrical conductivity measurements. Nevertheless, the knowledge of the surface adsorption properties of the ions can help to get information about tangential ionic mobility. Ions adsorbed as inner-sphere surface complexes lose part of their hydration shell and can be adsorbed at the close proximity of the mineral surface (Sverjensky 2005; Hiemstra \&
Van Riemsdijk 2006). The tangential mobility of these ions can hence be considerably smaller in the Stern layer than in bulk water. Ions adsorbed as outer-sphere surface complexes keep their hydration shell and can be adsorbed further away from the mineral surface compared to inner-sphere surface complexes (Sverjensky 2005; Hiemstra \& Van Riemsdijk 2006). The tangential mobility of these ions in the Stern layer can hence be comparable to their mobility in bulk water.

By combining eqs (6)-(9) and (11)-(13), the complex surface conductivity of a spherical particle of diameter $d$ can be expressed as a function of the size of the particle, electrochemical properties of the mineral/water interface, angular frequency and volumetric density of the particle:

$\sigma_{s}^{*}(d, \omega)=\frac{4}{d}\left[ \pm \beta_{b} Q_{b}\left(\frac{i \omega \tau_{b}}{1+i \omega \tau_{b}}\right)+\sum_{i=1}^{N} e z_{i} B_{i}^{d} \Gamma_{i}^{d}\right]+i \omega \alpha \rho_{s} \varepsilon_{0}$.

The low-frequency (DC) and high-frequency surface conductivity can be deduced from eq. (14) in the limits $\omega \rightarrow 0$ and $\omega \rightarrow \infty$, respectively. We obtain (Leroy et al. 2008):

$\sigma_{s}^{0}=\frac{4}{d} \Sigma_{s}^{d}=\frac{4}{d} \sum_{i=1}^{N} e z_{i} B_{i}^{d} \Gamma_{i}^{d}$

$\sigma_{s}^{\infty}=\frac{4}{d}\left(\Sigma_{s}^{b}+\Sigma_{s}^{d}\right)=\frac{4}{d}\left[ \pm \beta_{b} Q_{b}+\sum_{i=1}^{N} e z_{i} B_{i}^{d} \Gamma_{i}^{d}\right]$,

which shows that only the diffuse layer contributes to the DC surface conductivity whereas both the Stern and diffuse layer contribute to the surface conductivity at high frequency.

\subsubsection{Parameters inferred from the surface complexation model}

The parameter $M$ characterizing the decrease of the relaxation time of the Stern layer by the diffuse layer, is calculated using (Lyklema et al. 1983):

$M=1+\frac{q Q_{b}}{k_{B} T C_{d}}$,

where $C_{d}$ is the differential capacitance of the diffuse layer (in $\mathrm{C} \mathrm{m} \mathrm{m}^{-2} \mathrm{~V}^{-1}$ ), which can be computed as a function of the ions concentrations in bulk water $C_{i}^{w}\left(\mathrm{in} \mathrm{mol} \mathrm{L}^{-1}\right)$ and electrical potential at the beginning of the diffuse layer $\varphi_{d}$ according to the following equation (Appendix B):

$$
C_{d}=-\frac{\partial Q_{d}}{\partial \varphi_{d}}=\sqrt{\frac{\varepsilon_{w}}{2 k_{B} T}} \frac{\sum_{i=1}^{N} q_{i} 1000 N_{A} C_{i}^{w} \exp \left(-\frac{q_{i} \varphi_{d}}{k_{B} T}\right)}{\sqrt{\sum_{i=1}^{N} 1000 N_{A} C_{i}^{w}\left[\exp \left(-\frac{q_{i} \varphi_{d}}{k_{B} T}\right)-1\right]}},
$$

where $Q_{d}$ is the surface charge density of the diffuse layer, $q_{i}=$ $\pm e z_{i}$ is the ion charge (' + ' stands for cations and '-' stands for anions).

The parameters relative to the electrochemical properties of the mineral/water interface $Q_{b}, \varphi_{d}$ and $\Gamma_{i}^{d}$ are computed using a BSM of the mineral/water interface (Leroy \& Revil 2004, 2009; Leroy et al. 2008; Vaudelet et al. 2011). The results of the BSM computations are showed in Section 3. The surface charge density of adsorbed ions in the Stern layer, $Q_{b}$, and the electrical potential at the beginning of the diffuse layer, $\varphi_{d}$, are directly computed by the BSM. 
Table 1. Nomenclature of the macroscopic material properties.

\begin{tabular}{lll}
\hline Symbol & Meaning & Unit \\
\hline$\sigma^{*}$ & Complex conductivity of the porous medium & $\mathrm{S} \mathrm{m}^{-1}$ \\
$\sigma_{s}^{*}$ & Complex conductivity of the particles & $\mathrm{S} \mathrm{m}^{-1}$ \\
$\sigma_{w}^{*}$ & Complex conductivity of bulk water & $\mathrm{S} \mathrm{m}^{-1}$ \\
$\sigma_{s}$ & Surface conductivity of the particle & $\mathrm{S} \mathrm{m}^{-1}$ \\
$\varepsilon_{s}$ & Surface dielectric permittivity of the particle & $\mathrm{F} \mathrm{m}^{-1}$ \\
$\omega$ & Angular frequency & $\mathrm{rad} \mathrm{s}^{-1}$ \\
$f$ & Frequency & $\mathrm{Hz}^{-3}$ \\
$\rho_{s}$ & Volumetric density of the particle & $\mathrm{kg} \mathrm{m}^{-3}$ \\
$\sigma_{w}$ & Conductivity of the bulk water & $\mathrm{S} \mathrm{m}^{-1}$ \\
$\varepsilon_{w}$ & Dielectric permittivity of the bulk water & $\mathrm{F} \mathrm{m}^{-1}$ \\
$\varepsilon_{r}$ & Relative dielectric permittivity of the bulk water & $\mathrm{Dimensionless}^{-1}$ \\
$\varepsilon_{0}$ & Dielectric permittivity of the vacuum & $\mathrm{F} \mathrm{m}^{-1}$ \\
$\phi$ & Porosity of the granular medium & Dimensionless \\
$m$ & Cementation exponent of the particles & Dimensionless \\
$F$ & Electrical formation factor of the porous medium & Dimensionless \\
$d$ & Particle diameter & $\mathrm{m}$ \\
$a$ & Particle radius & $\mathrm{m}$ \\
$f\left(d_{i}\right)$ & Particle size distribution & Dimensionless \\
\hline & &
\end{tabular}

The surface site density of adsorbed ions in the diffuse layer $\Gamma_{i}^{d}$ is given by (Hunter 1981; Lyklema 1991; Leroy et al. 2012):

$$
\begin{aligned}
& \Gamma_{i}^{d}=C_{i}^{w} \int_{x=0}^{x=2 \kappa^{-1}}\left[\mathrm{e}^{-\frac{q_{i} \varphi(x)}{k_{B} T}}-1\right] d x, \\
& \varphi(x)=\varphi_{d} \mathrm{e}^{-\kappa x}, \\
& \kappa=\sqrt{\frac{2 e^{2} 1000 N_{A} I}{\varepsilon_{w} k_{B} T}}, \\
& I=0.5 \sum_{i=1}^{N} z_{i}{ }^{2} C_{i}^{w},
\end{aligned}
$$

where $x$ is the distance of the ion from the beginning of the diffuse layer ( $d$-plane) (in $\mathrm{m}), \kappa^{-1}$ is the Debye length (in $\mathrm{m}$ ), $\varphi(x)$ is the electrical potential in the diffuse layer, and $I$ is the ionic strength (in mol L ${ }^{-1}$ ). Eq. (20) holds for small electrical potentials at the onset of the diffuse layer of magnitude $<k_{b} T / e$ but remains accurate for larger electrical potentials (Hunter 1981; Lyklema 1991; Revil \& Glover 1997; Leroy et al. 2011). As we are interested to interpret the complex conductivity experiment of Wu et al. (2010) involving pore water containing saline water $\left(I \cong 0.03 \mathrm{~mol} \mathrm{~L}^{-1}\right)$ where the diffuse layer is compressed and zeta potential is low, eq. (20) can be applied in our case to describe the electrical potential distribution in the diffuse layer. The zeta potential is defined as the electrical potential at the onset of the diffuse layer.

The parameters of the complex conductivity model are summarized in Tables 1 and 2. In the next section, a BSM of the calcite/water interface is presented to compute the surface charge density of the Stern layer $Q_{b}$ and the electrical potential at the beginning of the diffuse layer $\varphi_{d}$.

\subsection{BSM of the calcite/water interface}

The BSM developed recently by Heberling et al. (2014) is used to describe the electrochemical properties of the Stern and diffuse layers of the calcite/water interface. The BSM considers that the Stern plane, that is the beta-plane, coincides with the $d$-plane corresponding to the onset of the diffuse layer, that is $\varphi_{b}=\varphi_{d}$ (Fig. 4). In our study, only the surface complexation reactions at the (lll $\left.\begin{array}{ll}1 & 0\end{array}\right)$

\begin{tabular}{|c|c|c|}
\hline Symbol & Meaning & Unit \\
\hline$\varphi$ & Electric potential in the diffuse layer & V \\
\hline$\varphi_{b}$ & Electric potential at the Stern layer & V \\
\hline$\varphi_{d}$ & $\begin{array}{l}\text { Electric potential at the beginning of the } \\
\text { diffuse layer }\end{array}$ & $\mathrm{V}$ \\
\hline$e$ & Elementary charge of the electron & $\mathrm{C}$ \\
\hline$N_{A}$ & Avogadro number & $\mathrm{mol}^{-1}$ \\
\hline$z_{i}$ & Ion valence & Dimensionless \\
\hline$q_{i}$ & Ion charge in the diffuse layer & $\mathrm{C}$ \\
\hline$q$ & Averaged ions charge in the Stern layer & $\mathrm{C}$ \\
\hline$C_{i}^{w}$ & Ion concentration in the bulk water & $\mathrm{mol} \mathrm{dm}{ }^{-3}$ \\
\hline$k_{B}$ & Boltzmann constant & $\mathrm{J} \mathrm{K}^{-1}$ \\
\hline$T$ & Temperature & $\mathrm{K}$ \\
\hline$I$ & Ionic strength & $\mathrm{mol} \mathrm{dm} \mathrm{m}^{-3}$ \\
\hline$\kappa$ & Inverse of the Debye length & $\mathrm{m}^{-1}$ \\
\hline$\Gamma_{i}^{d}$ & $\begin{array}{l}\text { Surface site density of adsorbed ions in the } \\
\text { diffuse layer }\end{array}$ & $\mathrm{m}^{-2}$ \\
\hline$Q_{b}$ & $\begin{array}{l}\text { Surface charge density of adsorbed ions in } \\
\text { the Stern layer }\end{array}$ & $\mathrm{C} \mathrm{m}^{-2}$ \\
\hline$Q_{d}$ & $\begin{array}{l}\text { Surface charge density of adsorbed ions in } \\
\text { the diffuse layer }\end{array}$ & $\mathrm{C} \mathrm{m}^{-2}$ \\
\hline$C_{d}$ & Differential capacitance of the diffuse layer & $\mathrm{C} \mathrm{m}^{-2} \mathrm{~V}^{-1}$ \\
\hline$\beta_{i}^{w}$ & Ion mobility in the bulk pore water & $\mathrm{m}^{2} \mathrm{~s}^{-1} \mathrm{~V}^{-1}$ \\
\hline$B_{i}^{d}$ & Ion effective mobility in the diffuse layer & $\mathrm{m}^{2} \mathrm{~s}^{-1} \mathrm{~V}^{-1}$ \\
\hline$\beta_{b}$ & Averaged ion mobility in the Stern layer & $\mathrm{m}^{2} \mathrm{~s}^{-1} \mathrm{~V}^{-1}$ \\
\hline$D_{b}$ & Averaged ion diffusivity in the Stern layer & $\mathrm{m}^{2} \mathrm{~s}^{-1}$ \\
\hline$\eta_{w}$ & Dynamic viscosity of bulk water & Pa s \\
\hline$\Sigma_{s}$ & $\begin{array}{l}\text { Total specific surface conductivity of the } \\
\text { particle }\end{array}$ & $\mathrm{S}$ \\
\hline$\Sigma_{s}^{b}$ & $\begin{array}{l}\text { Specific surface conductivity of the Stern } \\
\text { layer }\end{array}$ & S \\
\hline$\Sigma_{s}^{d}$ & $\begin{array}{l}\text { Specific surface conductivity of the diffuse } \\
\text { layer }\end{array}$ & S \\
\hline$\tau_{b}$ & $\begin{array}{l}\text { Relaxation time of the polarization of the } \\
\text { Stern layer }\end{array}$ & $\mathrm{s}$ \\
\hline$f_{b}$ & $\begin{array}{l}\text { Critical frequency of the polarization of the } \\
\text { Stern layer }\end{array}$ & $\mathrm{Hz}$ \\
\hline$M$ & $\begin{array}{l}\text { Diffuse layer polarization effects on Stern } \\
\text { layer polarization }\end{array}$ & Dimensionless \\
\hline
\end{tabular}
calcite surface, which is the dominating crystallographic plane on
Table 2. Nomenclature of the parameters at the scale of the electrical double layer.

most types of calcite, are considered. The total surface site density of calcium and carbonate surface sites on the calcite $\left(\begin{array}{lll}1 & 0 & 4\end{array}\right)$ face was estimated to be equal to 4.95 sites per $\mathrm{nm}^{-2}$ for each type of site using crystallographic studies (Wolthers et al. 2008; Heberling et al. 2011, 2014). According to Heberling et al. (2014) protonation only occurs for the calcium surface sites; calcite surface functional groups are hence controlled by the $>\mathrm{CaOH}_{2}^{0.5},>\mathrm{CaOH}^{-0.5}$ and $>\mathrm{CO}_{3}^{-0.5}$ surface sites.

The calcite surface is defined by the position of the surface $\mathrm{Ca}^{2+}$ ions (Vancappellen et al. 1993) and the 0-plane, where adsorption of protons occurs, is considered to be located a few Angströms (between $1.2 \AA$, Wolthers et al. 2008 and $2.3 \AA$, Heberling et al. 2014) from the calcite surface (Fig. 4). According to Heberling et al. (2011), the Stern plane, that is the beta-plane, is located beyond the two hydration water layers at a distance of 4-6 $\AA$ from the calcite surface. Therefore, counter-ions in the Stern layer are assumed to be mostly adsorbed as outer-sphere surface complexes at a distance of a few Angströms from the 0-plane (Stipp 1999). Furthermore, Heberling et al. (2014) assumed that the surface charge of their calcite samples is negative as a whole in their investigated $\mathrm{pH}$ range [5.5 10.5]. The surface charge of calcite was also observed to be negative in this $\mathrm{pH}$ range (Eriksson et al. 2007). Therefore, for the 


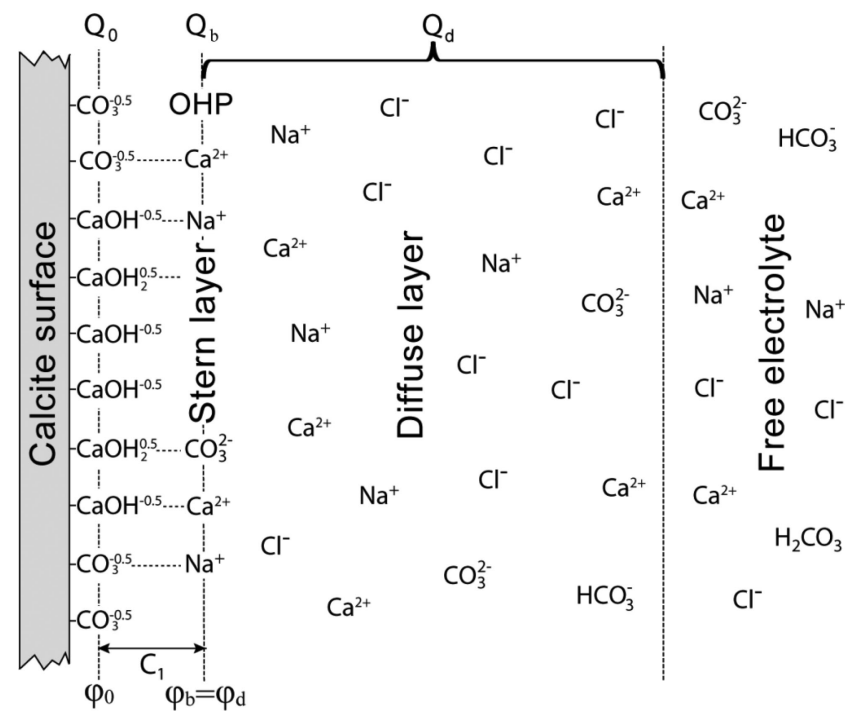

Figure 4. The basic Stern model used by Heberling et al. (2014) to describe the calcite/water interface (calcite $\left(\begin{array}{lll}1 & 0 & 4\end{array}\right)$ surface) when calcite is in contact with a $\mathrm{NaCl}$ and $\mathrm{CaCl}_{2}$ aqueous solution at equilibrium with a $\mathrm{pCO}_{2}$. The parameters $\varphi$ and $Q$ are the electrical potential and surface charge density, respectively, at the 0 -plane, $b$-plane (Stern plane) and $d$-plane (onset of the diffuse layer). According to the basic Stern model, the onset of the diffuse layer coincides with the Stern plane, that is $\varphi_{b}=\varphi_{d}$. OHP is the Outer Helmholtz plane. The parameter $C_{1}$ is the capacitance to model the electrical potential behaviour between the 0 -plane and $b$-plane.

$\mathrm{pH}$ conditions of the complex conductivity experiment of Wu et al. (2010) (basic pH), the calcite surface charge is compensated by hydrated cations in higher concentrations in the Stern layer than hydrated anions.

A geochemical code written in Matlab was developed to compute the surface complexation model of Heberling et al. (2014) (Li et al. 2016). The details of the calculation procedure are written in $\mathrm{Li}$ et al. (2016). The program combines aqueous complexation, surface complexation equilibria, surface charge density and mass balance conditions (Vancappellen et al. 1993; Hiemstra \& VanRiemsdijk 1996). The set of equations obtained is solved iteratively by the classical Newton-Raphson technique (Press et al. 1989). Table 3 summarizes, in matrix form, the set of equations relative to the calculation of surface and solution speciation for fixed values of $\mathrm{pH}$ and partial pressure of $\mathrm{CO}_{2}\left(\mathrm{pCO}_{2}\right)$. The value of the capacitance $C_{1}$ between the 0-plane and beta-plane is equal to $1.24 \mathrm{~F} \mathrm{~m}^{-2}(\mathrm{Li}$ et al. 2016).

In Section 3, our combined complex conductivity and surface complexation models will be used to interpret SIP experiments carried out by Wu et al. (2010) on glass beads pack where calcite precipitated at the glass beads surface. A sensitivity analysis of the petrophysical and interfacial parameters of our models will also be performed in Section 3.

\section{COMPARISON WITH EXPERIMENTAL DATA}

\subsection{Complex conductivity measurements of Wu et al. (2010)}

Wu et al. (2010) monitored calcite precipitation in glass beads pack using complex conductivity measurements in the frequency range [0.1-10000 Hz] under controlled laboratory conditions. The porous medium consisted of smooth glass beads of mean diameter of $3 \mathrm{~mm}$ packed in a transparent plexiglass column $8.4 \mathrm{~cm}$ long and $2.54 \mathrm{~cm}$ wide. The measured porosity was equal to 30 per cent. Wu et al. (2010) mixed two aqueous electrolytes, one containing $\mathrm{CaCl}_{2}$ at a concentration of 26.2 millimol L ${ }^{-1}(\mathrm{mM})$ (water electrical conductivity of $0.635 \mathrm{~S} \mathrm{~m}^{-1}$ ), and the other containing $\mathrm{Na}_{2} \mathrm{CO}_{3}$ at a concentration of $29 \mathrm{mM}$ (water electrical conductivity of $0.567 \mathrm{~S} \mathrm{~m}^{-1}$ ) at a fixed $\mathrm{pH}$ value comprised between 9 and 10 to favour calcite precipitation in glass beads column $\left[\mathrm{Ca}^{2+}+\mathrm{CO}_{3}^{2-} \Leftrightarrow \mathrm{CaCO}_{3}(s)\right]$. The saline $\mathrm{CaCl}_{2}$ solution was injected through the bottom of the column to establish an equilibrated baseline state. Following this, a second stream of $\mathrm{Na}_{2} \mathrm{CO}_{3}$ solution was introduced into the column from the injection port at the middle section to initiate calcite precipitation. The ionic concentrations were diluted once inside the column due to the equal volume mixing of these two solutions (initial ionic concentrations divided by two). The flow rate of both solutions was kept at $36 \mu \mathrm{L} \mathrm{min}{ }^{-1}$ for the duration of the experiment, which continued for 12 days past injection of $\mathrm{Na}_{2} \mathrm{CO}_{3}$. In the

Table 3. Stoichiometric matrix of aqueous and surface reactions at the calcite/water interface. The parameters $\varphi$ and $K$ are the electrical potentials at the different planes and the equilibrium constants of the reactions, respectively (adapted from the database Phreeqc.dat of the geochemical software Phreeqc (Parkhurst \& Appelo 2013) and from Heberling et al. 2014).

\begin{tabular}{lrrrrrrrrrr}
\hline Product species & $\mathrm{H}^{+}$ & $\mathrm{Cl}^{-}$ & $\mathrm{Na}^{+}$ & $\mathrm{Ca}^{2+}$ & $\mathrm{HCO}_{3}^{-}$ & $>\mathrm{CaOH}^{-0.5}$ & $>\mathrm{CO}_{3}^{-0.5}$ & $\mathrm{e}^{\frac{-e \varphi_{0}}{k_{B} T}}$ & $\mathrm{e}^{\frac{-e \varphi_{\beta}}{k_{B} T}}$ & $\log _{10} K$ \\
\hline $\mathrm{CO}_{3}^{-2}$ & -1 & 0 & 0 & 0 & 1 & 0 & 0 & 0 & 0 & -10.33 \\
$\mathrm{H}_{2} \mathrm{CO}_{3}$ & 1 & 0 & 0 & 0 & 1 & 0 & 0 & 0 & 0 & 6.35 \\
$\mathrm{CaHCO}_{3}^{+}$ & 0 & 0 & 0 & 1 & 1 & 0 & 0 & 0 & 0 & 1.11 \\
$\mathrm{CaCO}_{3}(\mathrm{aq})$ & -1 & 0 & 0 & 1 & 1 & 0 & 0 & 0 & 0 & -7.10 \\
$\mathrm{CaOH}^{+}$ & -1 & 0 & 0 & 1 & 0 & 0 & 0 & 0 & 0 & -12.78 \\
$>\mathrm{CaOH}_{2}^{+0.5}$ & 1 & 0 & 0 & 0 & 0 & 1 & 0 & 1 & 0 & 0.50 \\
$>\mathrm{CaOH}_{2}^{+0.5} \ldots \mathrm{Cl}^{-}$ & 1 & 1 & 0 & 0 & 0 & 1 & 0 & 1 & -1 & 0.45 \\
$>\mathrm{CaOH}^{-0.5} \ldots \mathrm{Na}^{+}$ & 0 & 0 & 1 & 0 & 0 & 1 & 0 & 0 & 1 & 0.56 \\
$>\mathrm{CaOH}^{-0.5} \ldots \mathrm{Ca}^{2+}$ & 0 & 0 & 0 & 1 & 0 & 1 & 0 & 0 & 2 & 1.68 \\
$>\mathrm{CaOH}_{2}^{+0.5} \ldots \mathrm{HCO}_{3}^{-}$ & 1 & 0 & 0 & 0 & 1 & 1 & 0 & 1 & -1 & 0.54 \\
$>\mathrm{CaOH}_{2}^{+0.5} \ldots \mathrm{CO}_{3}^{2-}$ & 0 & 0 & 0 & 0 & 1 & 1 & 0 & 1 & -2 & -6.57 \\
$>\mathrm{CO}_{3} \mathrm{H}^{+0.5}$ & 1 & 0 & 0 & 0 & 0 & 0 & 1 & 1 & 0 & -20.00 \\
$>\mathrm{CO}_{3}^{-0.5} \ldots \mathrm{Na}^{+}$ & 0 & 0 & 1 & 0 & 0 & 0 & 1 & 0 & 1 & 0.56 \\
$>\mathrm{CO}_{3}^{-0.5} \ldots \mathrm{Ca}^{2+}$ & 0 & 0 & 0 & 1 & 0 & 0 & 1 & 0 & 2 & 1.68 \\
\hline
\end{tabular}


complex conductivity experiment of Wu et al. (2010), sodium ion is the dominating cation in solution because $C_{\mathrm{Na}^{+}}^{w}=29 \mathrm{mM}$ whereas $C_{\mathrm{Ca}^{2+}}^{w}<2 \mathrm{mM}$ after the first stage of calcite precipitation (two days after the beginning of the experiment).

The complex conductivity in the column was monitored by $\mathrm{Wu}$ et al. (2010) using a four $\mathrm{Ag} / \mathrm{AgCl}$ electrodes system with a Wenner$\alpha$ configuration (the two measuring potential electrodes are located between the two injecting current electrodes) and a NI 4461 board for the data acquisition (National Instruments). Using the injected calcium ion concentration, the measured calcium ion concentration in the effluent, and the flow rate, Wu et al. (2010) estimated a constant precipitation rate of $0.6 \mathrm{mM} \mathrm{CaCO}_{3}$ per day. Furthermore, Wu et al. (2010) used a Cole-Cole model to interpret their complex conductivity measurements and fitted a mean relaxation time $\tau$ and chargeability $m_{n}\left(m_{n}=\left(\sigma_{\infty}-\sigma_{0}\right) / \sigma_{\infty}\right.$ Weller et al. 2013) as a function of time (in days). They also performed complementary photographic and scanning electron microscopy imaging. Wu et al. (2010) observed two distinct phases of calcite precipitation onto the glass beads. During the first phase of the calcite precipitation experiment ( 9 days after the beginning of the experiment), Wu et al. (2010) observed a decreasing mean characteristic frequency and increasing quadrature conductivity with time, and they estimated an increasing mean relaxation time and chargeability with time. During the second phase of the calcite precipitation experiment (912 days after the beginning of the experiment), Wu et al. (2010) observed an opposite behaviour of the measured quadrature conductivity, that is an increasing mean characteristic frequency and decreasing quadrature conductivity with time, and they estimated a decreasing mean relaxation time and chargeability with time. They observed an earlier phase of calcite precipitation characterized by well distributed and discrete calcite particles growing with time around individual glass beads (limited deposition in the pore spaces) and a later phase of calcite precipitation characterized by calcite aggregates starting to fill the pore spaces between glass beads coated by calcite.

Nevertheless, Wu et al. (2010) used complementary photographic and scanning electron microscopy imaging techniques to get information on the evolution of the size and shape of precipitated calcite particles. Their Cole-Cole model gives limited information on the evolution of the textural and interfacial properties of their glass beads pack. Furthermore, Wu et al. (2010) suggested that the fixed layer polarization mechanism can explain their observations but they did not use a mechanistic model of Stern layer polarization to explain their SIP data. In the following section, we will use our mechanistic Stern layer polarization model to get information on the evolution of the size and shape of precipitating calcite particles and to describe the electrochemical properties of the calcite/water interface responsible for the measured spectral induced polarization response. It should be noted that the SIP measurements of Wu et al. (2010) may present significant uncertainties in the high frequency range [1000-10 $000 \mathrm{~Hz}$ ] because measured voltage was not corrected of impedances for potential electrodes (Huisman et al. 2015).

\subsection{Complex conductivity modelling}

\subsubsection{Modelling strategy}

The grain polarization model based on the electrochemical polarization of the Stern layer of calcite is used to model induced polarization of calcite precipitation on glass beads. The complex conductivity model was shown to be insensitive to the complex surface conductivity of millimetric glass beads because their size is considerably larger than the size of micrometric calcite particles (surface conductivity decreases when size of the particle increases, eq. 7). Furthermore, before calcite precipitation (at day 0 of the experiment), comparably small polarization of the porous sample was observed during the complex conductivity experiment of $\mathrm{Wu}$ et al. (2010). Frequency evolution of measured imaginary conductivity (sensitive to polarization) of glass beads pack was seen to be negligible at day 0 (see their fig. $4 a$ ). In addition, the contribution of glass beads to the DC surface conductivity of glass beads pack can also be neglected. Indeed, the electrical formation factor of smooth glass beads of mean diameter $3 \mathrm{~mm}$ (the parameter $F$ in eq. 1) was estimated to be equal to 6.08 using a measured porosity $\phi$ value of 0.3 and a cementation exponent $m$ equal to 1.5 corresponding to spherical particles (Sen et al. 1981) $\left(F=\phi^{-m}\right.$, eq. 2 ). Using $F=6.08$, the measured DC conductivity of bulk water ( $\sigma_{w} \cong 635 \mathrm{mS} \mathrm{m}^{-1}$; Wu et al. 2010) and of the glass beads pack before calcite precipitation $\left(\sigma \cong 110 \mathrm{mS} \mathrm{m}^{-1}\right.$; Wu et al. 2010), we found that the surface conductivity of glass beads can be neglected compared to the water conductivity, as expected by $\mathrm{Wu}$ et al. (2010). Therefore, only the surface conductivity of calcite particles is considered for the calculation of $\sigma_{s}^{*}$ in eq. (1). Furthermore, only the cementation exponent of calcite particles is considered in our conductivity modelling. Nevertheless, glass beads control the porosity of the porous medium that is used in our conductivity modelling through the use of the electrical formation factor (eq. 1).

The surface charge density of the Stern layer, $Q_{b}$, and the electrical potential at the onset of the diffuse layer of the calcite/water interface, $\varphi_{d}$, were computed as a function of the known chemical composition of the pore water using our BSM. The surface site densities of the ions in the diffuse layer, $\Gamma_{i}^{d}$, were computed using eqs (19)-(22). The differential capacitance of the diffuse layer, $C_{d}$, was computed as a function of $\varphi_{d}$ using eq. (18). The $M$ parameter was calculated according to $C_{d}$ and $Q_{b}$ using eq. (17). During the complex conductivity experiment of Wu et al. (2010), the chemical composition of bulk water was roughly constant between day 2 and day 12 after the beginning of the experiment when polarization is observed. Therefore, the values of the electrochemical properties of the calcite/water interface were taken constant in our modelling. These values are reported in Table 4 .

The value of the $M$ parameter characterizing the effects of the polarized diffuse layer on the Stern layer polarization is equal to 30.7 (eq. 17). This value is significantly higher than the $M$ values reported in Niu et al. (2016), being comprised between 1 and 3. Nevertheless, Niu et al. (2016) did not use a surface complexation model of the calcite/water interface to compute $M$. In addition, for a given relaxation time $\tau_{b}$, a higher $M$ value induces a smaller diffusion coefficient in the Stern layer $D_{b}$ and/or larger particles size $d$ according to eq. (10). These parameters values are more realistic than the parameters values deduced from $M=1$. Furthermore, it should be noted that even if the computed surface site densities of ions in the diffuse layer are low compared to the computed surface site densities of adsorbed ions in the Stern layer (Table 4), the effects of diffuse layer polarization on Stern layer polarization cannot be ignored. The double layer as a whole is always approximately electroneutral and the deviation of the charge of the polarized diffuse layer exactly cancels the deviation of the charge of the polarized Stern layer (eq. B8; Lyklema et al. 1983).

The complex surface conductivity of calcite particles of size $d$, $\sigma_{s}^{*}(d)$, was calculated according to the electrochemical properties of the calcite/water interface using eqs (10), (13) and (14). The 
Table 4. Modelled electrochemical properties of the calcite/water interface.

\begin{tabular}{|c|c|}
\hline Parameters & Values \\
\hline $\mathrm{Na}^{+}$concentration in bulk water $(\mathrm{mM})$ & 29.00 \\
\hline $\mathrm{Ca}^{2+}$ concentration in bulk water $(\mathrm{mM})$ & 1.20 \\
\hline $\mathrm{Cl}^{-}$concentration in bulk water $(\mathrm{mM})$ & 29.00 \\
\hline $\mathrm{CO}_{3}^{2-}$ concentration in bulk water $(\mathrm{mM})$ & 0.09 \\
\hline $\mathrm{HCO}_{3}^{-}$concentration in bulk water $(\mathrm{mM})$ & 1.20 \\
\hline $\mathrm{pH}$ & 9.00 \\
\hline Surface charge density of the Stern layer $Q_{b}\left(\mathrm{C} \mathrm{m}^{-2}\right)$ & 0.56 \\
\hline Surface site density of adsorbed $\mathrm{Na}^{+}$in the Stern layer $\left(10^{17}\right.$ sites $\left.\mathrm{m}^{-2}\right)$ & 17.30 \\
\hline Surface site density of adsorbed $\mathrm{Ca}^{2+}$ in the Stern layer $\left(10^{17}\right.$ sites $\left.\mathrm{m}^{-2}\right)$ & 8.91 \\
\hline Surface charge density of the diffuse layer $Q_{d}\left(\mathrm{C} \mathrm{m}^{-2}\right)$ & 0.012 \\
\hline Surface site density of adsorbed $\mathrm{Na}^{+}$in the diffuse layer $\left(10^{17}\right.$ sites $\left.\mathrm{m}^{-2}\right)$ & 0.383 \\
\hline Surface site density of adsorbed $\mathrm{Ca}^{2+}$ in the diffuse layer $\left(10^{17}\right.$ sites $\left.\mathrm{m}^{-2}\right)$ & 0.032 \\
\hline Surface site density of adsorbed $\mathrm{Cl}^{-}$in the diffuse layer $\left(10^{17}\right.$ sites $\left.\mathrm{m}^{-2}\right)$ & 0.189 \\
\hline Surface site density of adsorbed $\mathrm{CO}_{3}^{2-}$ in the diffuse layer $\left(10^{17}\right.$ sites $\left.\mathrm{m}^{-2}\right)$ & 0.0005 \\
\hline Surface site density of adsorbed $\mathrm{HCO}_{3}^{-}$in the diffuse layer $\left(10^{17}\right.$ sites $\left.\mathrm{m}^{-2}\right)$ & 0.033 \\
\hline Electrical potential at the beginning of the diffuse layer $\varphi_{d}(\mathrm{mV})$ & -29.00 \\
\hline Differential capacitance of the diffuse layer $C_{d}\left(\mathrm{C} \mathrm{m}^{-2} \mathrm{~V}^{-1}\right)$ & 0.69 \\
\hline Diffuse layer polarization effects on Stern layer polarization $M$ & 30.70 \\
\hline
\end{tabular}

volumetric density of calcite, $\rho_{s}$, is equal to $2710 \mathrm{~kg} \mathrm{~m}^{-3}$ (Wolthers et al. 2012). In eq. (10), the tangential mobility of the counterions in the Stern layer of the calcite/water interface, $\beta_{b}$, remains unknown (Ricci et al. 2013). Cations are assumed to be mostly adsorbed in the Stern layer of calcite as outer-sphere surface complexes because, similarly to the silica/water interface (Sverjensky 2001; Vaudelet et al. 2011; Leroy et al. 2013), the large hydration layer at the calcite surface repels hydrated cations from the surface (Stipp 1999; Heberling et al. 2011, 2014). Nevertheless, as pointed out by Wolthers et al. (2008) and Ricci et al. (2013), some $\mathrm{Na}^{+}$and $\mathrm{Ca}^{2+}$ counter-ions may also be adsorbed as inner-sphere surface complexes. It results that $\mathrm{Na}^{+}$and $\mathrm{Ca}^{2+}$ counter-ions may be less mobile in the Stern layer than in bulk water and, following the recent molecular dynamics simulations and atomic force microscopy (AFM) measurements of Ricci et al. (2013), we choose a mobility ratio of 0.1 between ions adsorbed in the Stern layer and ions in bulk water. We took a value of $5.7 \times 10^{-9} \mathrm{~m}^{2} \mathrm{~s}^{-1} \mathrm{~V}^{-1}$ for the ion mobility in the Stern layer. This value is the mobility ratio times the arithmetic average of the ion mobility of $\mathrm{Na}^{+}$and $\mathrm{Ca}^{2+}$ in dilute water for a temperature of $298 \mathrm{~K}\left(25^{\circ} \mathrm{C}\right)\left(\beta_{\mathrm{Na}^{+}}^{w}=5.18 \times 10^{-8}\right.$ and $\beta_{\mathrm{Ca}^{2+}}^{w}=6.18 \times 10^{-8} \mathrm{~m}^{2} \mathrm{~s}^{-1} \mathrm{~V}^{-1}$, Parkhurst \& Appelo 2013). The values of the ions mobilities in bulk water are presented in Table 5. In eq. (14), the effective ion mobility in the diffuse layer, $B_{i}^{d}$, was calculated according to the ion mobility in bulk water and considering electro-osmosis (eq. 13).

The complex surface conductivity of one calcite particle of diameter $d_{i}, \sigma_{s}^{*}\left(d_{i}\right)$, was weighted by its relative volume in the solid, that is by the discretized particle size distribution $f\left(d_{i}\right)$. The complex surface conductivity of calcite particles of different sizes, $\sigma_{s}^{*}$, was computed by adding the weighted contributions of the calcite particles of different diameters (eqs 4 and 5). Furthermore, the complex conductivity of bulk water $\sigma_{w}^{*}$ was computed as a function of the measured water conductivity and frequency according to eq. (3).
Table 6. Parameters of our complex conductivity model.

\begin{tabular}{lc}
\hline Parameters & Values \\
\hline Ion mobility in the Stern layer, $\beta_{b}\left(\mathrm{~m}^{2} \mathrm{~s}^{-1} \mathrm{~V}^{-1}\right)$ & $5.7 \times 10^{-9}$ \\
Initial cementation exponent of the particles, $m$ & 1.35 \\
Initial glass beads porosity, $\phi$ & 0.30 \\
Bulk pore water conductivity, $\sigma_{w}\left(\mathrm{~S} \mathrm{~m}^{-1}\right)$ & 0.356 \\
\hline
\end{tabular}

The water conductivity of the glass beads pack was measured by $\mathrm{Wu}$ et al. (2010) as a function of time (days) after the beginning of the experiment and was seen to be roughly constant after 2 days of experiment ( $\sigma_{w} \cong 356 \mathrm{mS} \mathrm{m}^{-1}$; Wu et al. 2010). The resulting complex surface conductivity of calcite particles of different sizes $\sigma_{s}^{*}$ and the modelled complex conductivity of the bulk water $\sigma_{w}^{*}$ (eq. 3) were introduced in the DEM model to compute the complex conductivity of the glass beads pack (eqs 1 and 2). The cementation exponent of the calcite particles and the porosity of the glass beads pack were taken initially as $m=1.35$ and $\phi=0.3$, respectively. The cementation exponent of dispersed calcite crystals is typically between 1.3 and 1.5 (Guichet et al. 2006; Li et al. 2016). The parameter values of the complex conductivity model are summarized in Table 6. The porosity of the glass beads pack estimated according to Wu et al. (2010) is calculated considering the measured initial porosity of the glass beads pack and the volume of precipitated calcite estimated by Wu et al. (2010). Wu et al. (2010) reported $0.6 \mathrm{mM}$ of calcite precipitated per day, which corresponds to a volume of precipitated calcite of $0.023 \mathrm{~cm}^{3}$ per day using a molar mass of 100 $\mathrm{g}$ for $\mathrm{CaCO}_{3}$ and a calcite volumetric density of $2.71 \mathrm{~g} \mathrm{~cm}^{-3}$. As the total volume of the sample in the experiment of Wu et al. (2010) is equal to $42.5 \mathrm{~cm}^{3}$, the porosity is expected to decrease by 0.05 per cent each day.

The quadrature conductivity measurements of $\mathrm{Wu}$ et al. (2010) were inverted using the Matlab code developed by Florsch et al.

Table 5. Ionic mobilities in bulk water (in $10^{-8} \mathrm{~m}^{2} \mathrm{~s}^{-1} \mathrm{~V}^{-1}$; temperature $T=298 \mathrm{~K}$ ) from the Phreeqc database phreeqc.dat (Parkhurst \& Appelo 2013).

\begin{tabular}{lllllccc}
\hline Ion & $\mathrm{Na}^{+}$ & $\mathrm{H}^{+}$ & $\mathrm{Cl}^{-}$ & $\mathrm{OH}^{-}$ & $\mathrm{HCO}_{3}^{-}$ & $\mathrm{Ca}^{2+}$ & $\mathrm{CO}_{3}^{2-}$ \\
\hline$\beta_{i}^{w}$ & 5.18 & 36.25 & 7.90 & 20.52 & 4.60 & 6.18 & 7.44 \\
\hline
\end{tabular}



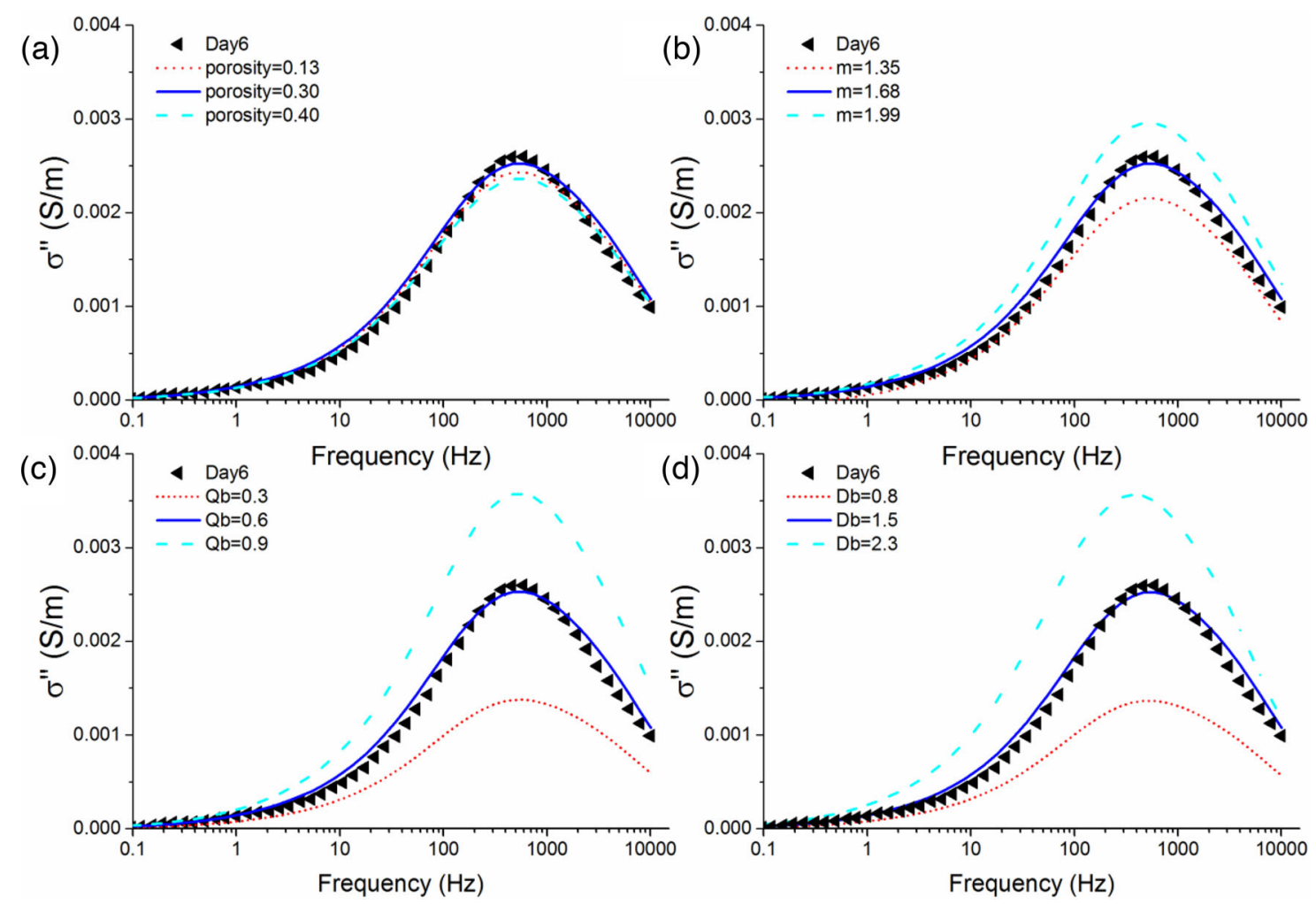

Figure 5. Effects of porosity of glass beads pack $\phi$ (a), cementation exponent $m$ (b), surface charge density of the Stern layer $Q_{b}$ (in $\mathrm{C} \mathrm{m}{ }^{-2}$ ) (c) and diffusion coefficient $D_{b}$ (in $10^{-10} \mathrm{~m}^{2} \mathrm{~s}^{-1}$ ) (d) on the modelled imaginary conductivity. The computations of the complex conductivity model are represented by the lines and the symbols represent the imaginary conductivity measurements of Wu et al. (2010).

(2014) to obtain the discretized relaxation time $f\left(\tau_{i}\right)$ and particle size distribution $f\left(d_{i}\right)$ (Revil \& Florsch 2010; Florsch et al. 2014; Niu et al. 2016). Florsch et al. (2014) used generalized relaxation basis functions (such as the generalized Cole-Cole function) and the L-curve approach to optimize the damping parameter required to get smooth and realistic inverse solutions (read Florsch et al. 2014 for further information relative to the inversion procedure). The particle sizes were computed from the surface mobility of the counter-ions in the Stern layer, the computed $M$ parameter and the inverted relaxation times $\left(d_{i}=\sqrt{8 k_{B} T \beta_{b} M \tau_{i} /|q|}\right.$, eq. 10). It should be therefore noted that the inverted particle size distribution $f(d)$ is dependent on the value chosen for $M$ and the ion mobility in the Stern layer $\beta_{b}$. Finally, only the cementation exponent $m$ of calcite particles was adjusted by the gradient method as a function of the days after the beginning of the experiment to match the real and imaginary conductivity measurements of Wu et al. (2010) $\left(\sigma^{*}=\sigma^{\prime}+i \sigma^{\prime \prime}\right.$ with $\sigma^{\prime}$ the real part and $\sigma^{\prime \prime}$ the imaginary part of the complex conductivity).

\subsubsection{Sensitivity analysis and results of the modelling}

A preliminary sensitivity analysis of the effects of petrophysical and interfacial parameters on the modelled imaginary conductivity was carried out. Different values of the porosity $\phi$ of glass beads pack, cementation exponent $m$ of calcite particles, surface charge density of the Stern layer $Q_{b}$ and tangential diffusion coefficient of counterions in the Stern layer $\beta_{b}$ were tested (Fig. 5). Compared to the other parameters, the modelled imaginary conductivity is not very sensitive to the porosity of glass beads pack. The modelled imaginary conductivity only slightly decreases when porosity increases due to the smaller calcite quantity (Fig. 5a), whereas it increases sharply when cementation exponent, surface charge density of the Stern layer or ions diffusion coefficient in the Stern layer increases (Figs $5 \mathrm{~b}-\mathrm{d}$ ). It should be noted that the characteristic frequencies shift towards lower frequencies when ions diffusion coefficient in the Stern layer increases because of the relationship between the relaxation time and the ions diffusion coefficient in the Stern layer, that is for the same inverted relaxation time, the size of the calcite particles must increase if ions diffusion coefficient in the Stern layer increases $\left(\tau_{b}=a^{2} / 2 D_{b} M\right.$, eq. 10$)$.

As calcite crystals continuously precipitate in glass beads pack, there may be some fluctuations of the surface charge density of the Stern layer of calcite crystals, which may be caused by the surface sites on the different surfaces of growing calcite crystals, even if calcite crystals are in contact with a stable electrolyte (Leroy \& Revil 2004; Leroy et al. 2006). However, in our models, the surface charge density of the Stern layer was not adjusted to match the imaginary conductivity data. Instead, the cementation exponent $m$ describing the shape of calcite particles is the adjusted parameter. A further experimental study is necessary to distinguish the surface charge effects from the textural parameters on the SIP response of calcite crystal growth in porous medium.

The evolution of the modelled imaginary conductivity as a function of frequency and time (in days) is in very good agreement with the measurements of $\mathrm{Wu}$ et al. (2010; Fig. 6). The evolution of the modeled particle size distribution during the calcite precipitation experiment is presented at Fig. 7. The smallest particles size information is missing due to lack of the complex conductivity measurements at high frequency $(>10 \mathrm{kHz})$. Before clogging (referred to phase 1 in Wu et al. 2010; at day 9), the modelled particles size increases as experiment continues. It is consistent with the visual 

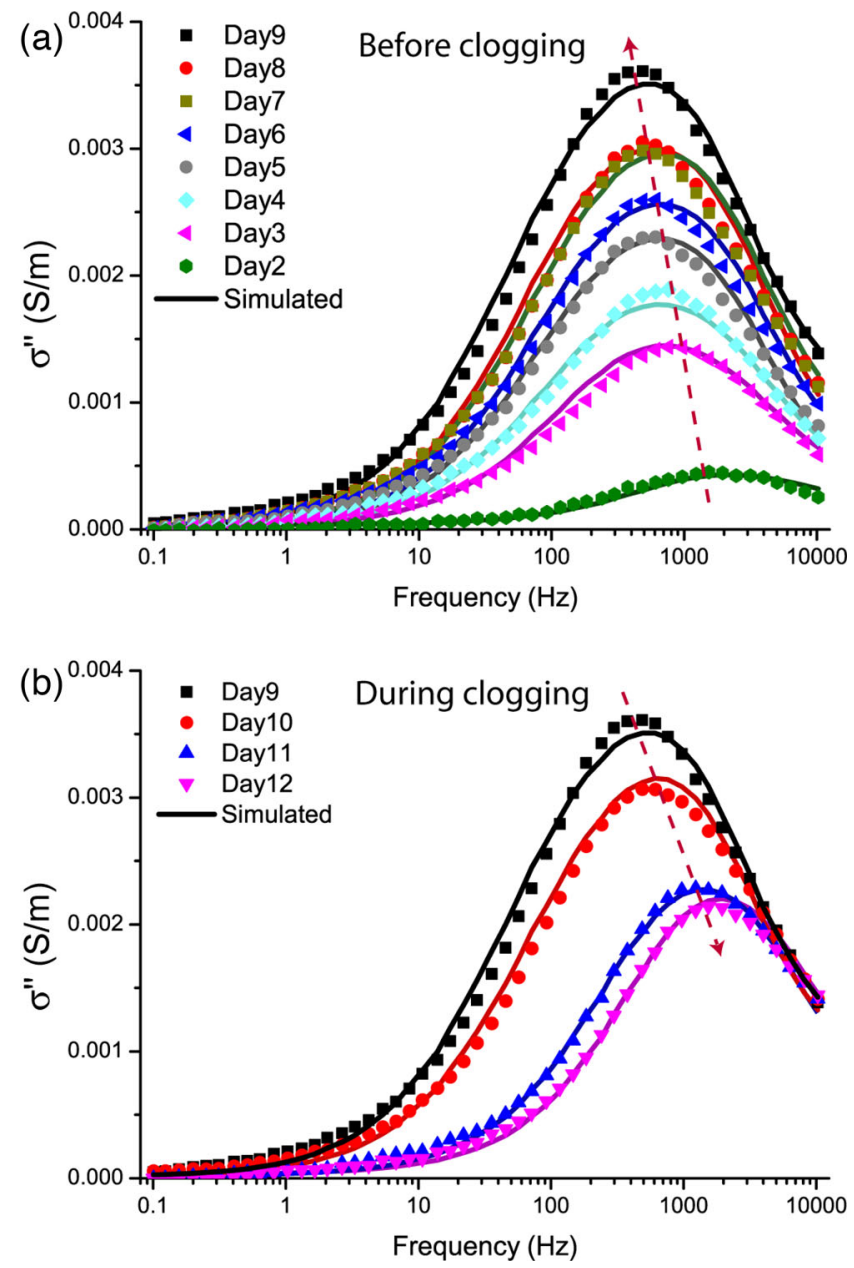

Figure 6. Imaginary conductivity spectra of calcite precipitation on glass beads as a function of time in days (after the beginning of the experiment) before the pore clogging by the calcite precipitates (a) and during the pore clogging by the calcite precipitates (b). Imaginary conductivities inferred from the complex conductivity model are represented by the lines and the symbols represent the imaginary conductivity measurements of $\mathrm{Wu}$ et al. (2010).

observations from scanning electron microscopy (SEM) images in the experiment ( $\mathrm{Wu}$ et al. 2010). The calcite particles increase approximately from less than 5 to $20 \mu \mathrm{m}$ (100 $\mu \mathrm{m}$ maximum), as reported by $\mathrm{Wu}$ et al. (2010). The adjusted cementation exponent and the formation factor increase with time during the first phase of calcite precipitation experiment, from 1.35 (day 2) to 1.9 (day 9) and 5 (day 2) to 13 (day 9), respectively (Fig. 8). The increase of the cementation exponent of calcite particles with time corresponds to the flattening of calcite particles, that is individual calcite crystals aggregate into larger but flatter calcite grains because calcite crystals grow laterally and come in contact with each other. Wu et al. (2010) observed flattened and aggregated calcite particles at the end of their calcite precipitation experiment (Fig. 8).

In the differential effective medium theory applied to granular porous media, the cementation exponent increases from 1.5 (spheres) to 4 (highly oblate particles) when the aspect ratio of the particle increases (Mendelson \& Cohen 1982). As calcite particles flatten during their precipitation on the glass beads surface, their aspect ratio increases and then their cementation exponent increases from 1.35 to 1.9 in our study. A value of 2 for the cementation exponent of the particles corresponds to the cementation exponent
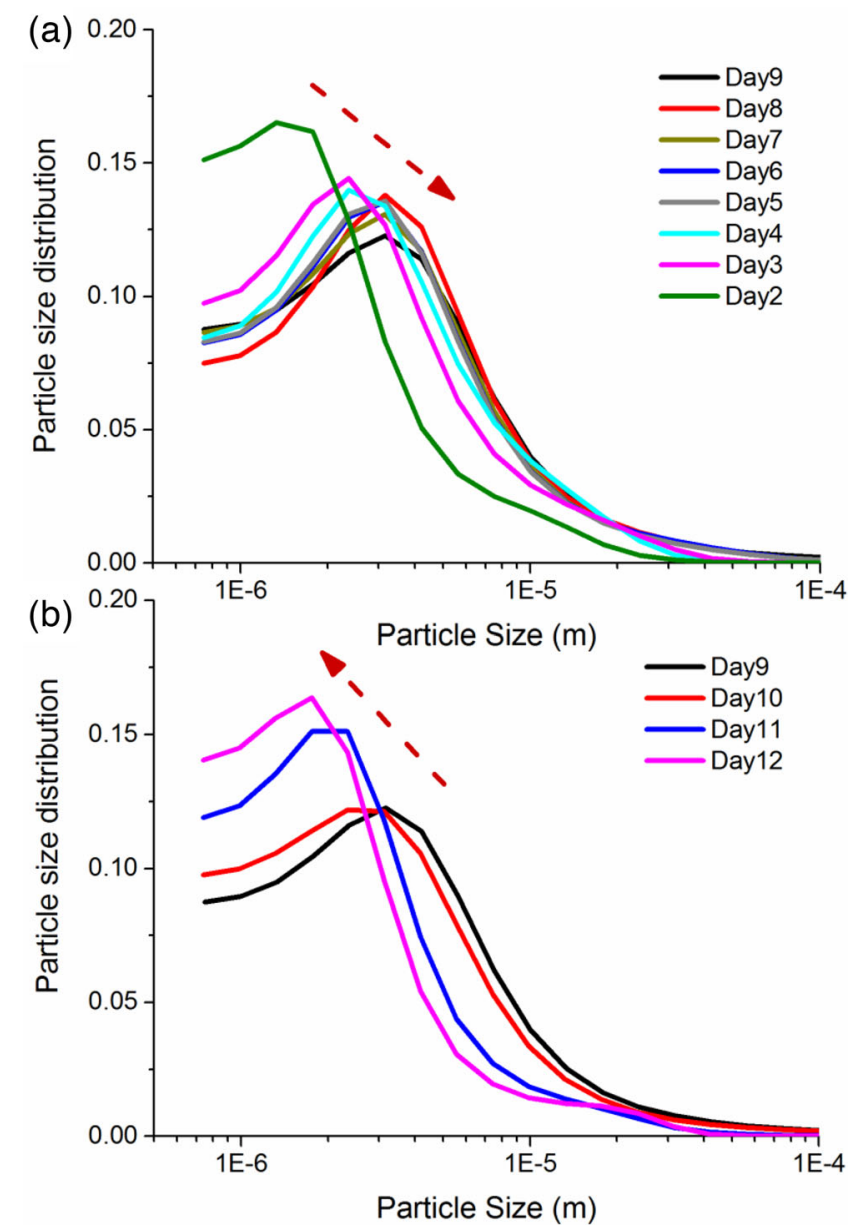

Figure 7. Evolution of the computed particle size distribution of calcite particles during calcite precipitation experiment obtained from the relaxation time distribution (RTD) code (Florsch et al. 2014).

value of sandstones (Revil et al. 1998). Calcite crystals are assumed to initially have a cementation exponent of 1.35 because they are rhombohedral and not spherical (Guichet et al. 2006). Cementation exponents of granular porous media lie in the range [1.2-4] (Mendelson \& Cohen 1982).

As the calcite precipitation experiment continued over 9 days, the clogging in the pore space between glass beads starts to occur in the sample holder because glass beads are entirely coated by calcite precipitates. Wu et al. (2010) observed using scanning electron microscopy that pores started to be occupied by precipitated calcite at the bottom of their sample holder at the end of their precipitation experiment (during the last 3 days of the experiment, fig. $6 \mathrm{~g}$ of $\mathrm{Wu}$ et al. 2010). At the second stage of the calcite precipitation experiment, the cementation exponent of calcite particles and the formation factor of glass beads pack decrease with time as shown in Fig. 8, from 1.9 to 1.65 and 13 to 10, respectively. The decrease of the cementation exponent of calcite particles with time suggests that more dispersed and smaller calcite crystals polarize as experiment continues. The modelled particle size distribution obtained from the inverted imaginary conductivity spectra moves towards smaller particles as experiment continues (Figs 6b and 7b). Large calcite aggregates spread over multiple glass beads during the clogging process and they do not play an important role in the measured complex conductivity spectra because they are too large to polarize in the investigated frequency range. Only new and 


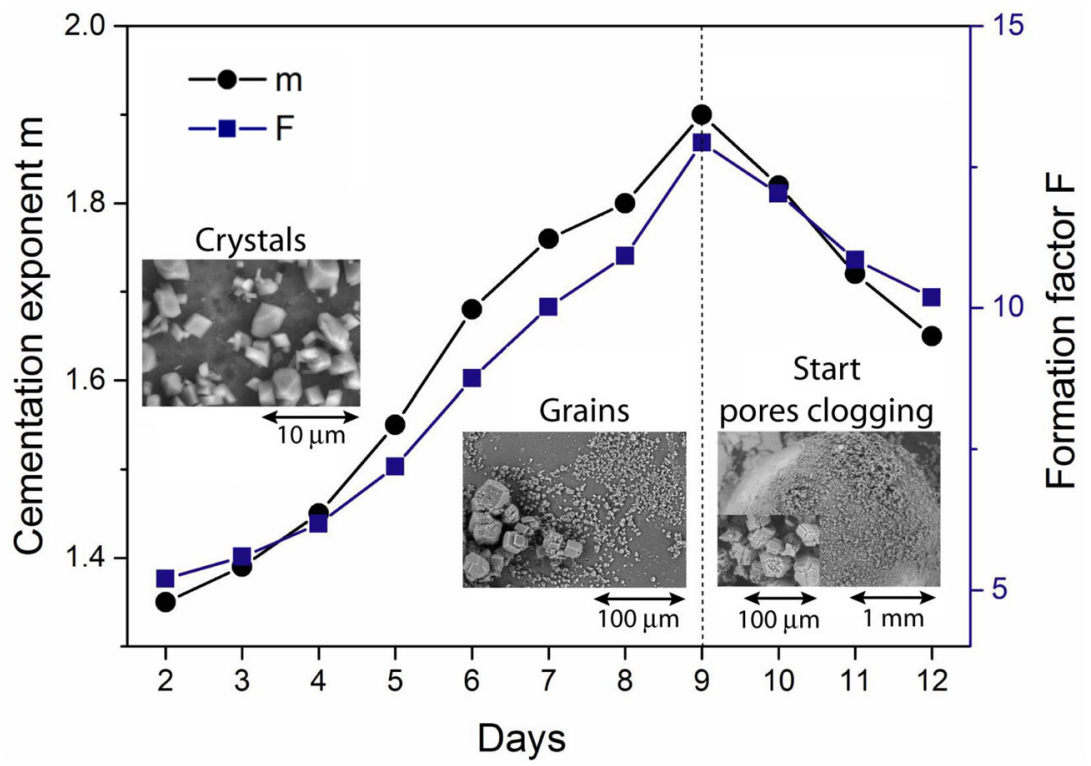

Figure 8. Computed particles cementation exponent $(m)$ and formation factor $F$ changes during the calcite precipitation experiment of Wu et al. (2010). The cementation exponent and formation factor of the glass beads pack where calcite precipitation occurs have a different trend of changes before and after the starting of the pore clogging (day 9).

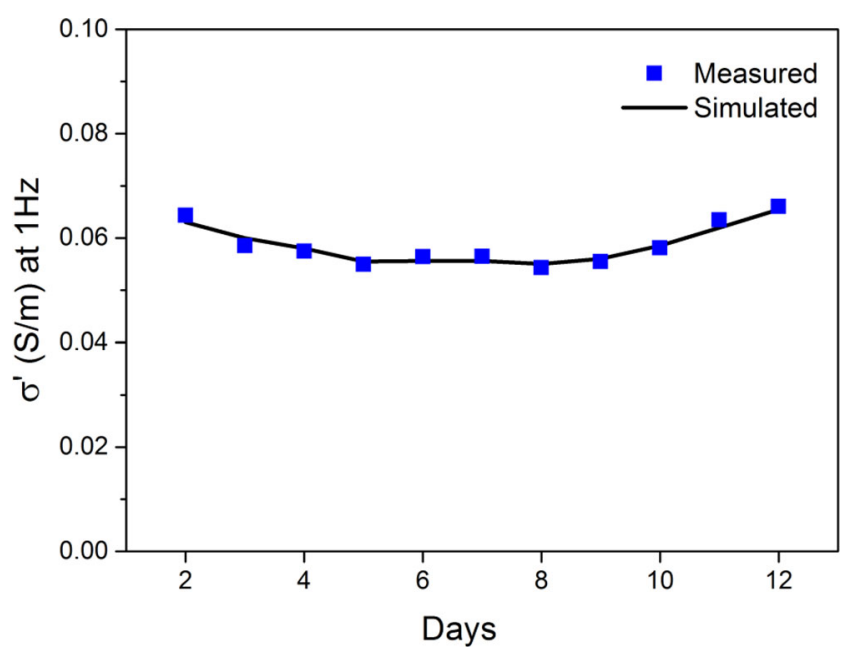

Figure 9. In-phase conductivity of the calcite precipitation experiment of Wu et al. (2010). In-phase conductivities inferred from the complex conductivity model are represented by the line and the symbols represent the real conductivity measurements of Wu et al. (2010).

remaining smaller calcite crystals contribute to the complex surface conductivity of glass beads pack at the second stage of the calcite precipitation experiment. Our complex conductivity model was also able to reproduce the in-phase conductivity data measured by $\mathrm{Wu}$ et al. (2010) from day 2 to day 12 after the beginning of the experiment (Fig. 9). The sample conductivity is roughly constant from day 2 to day 12 because the decreasing in-phase conductivity due to the increasing formation factor is compensated by the increasing surface conductivity of calcite particles from day 2 to day 9 and the decreasing surface conductivity of calcite particles is compensated by the increasing in-phase conductivity due to the decreasing formation factor from day 9 to day 12 .

Our estimation of the evolution of the size and shape of calcite particles precipitating on glass beads according to SIP measurements and mechanistic models can be useful to monitor the trans- port properties such as the permeability of sandstones containing saline solutions favouring calcite precipitation. Indeed, permeability can be estimated according to the particle size and shape (Revil \& Cathles 1999; Revil \& Florsch 2010). We also showed that the Stern layer of calcite controls the measured imaginary conductivity response of glass beads pack reported in Wu et al. (2010) and that the diffuse layer polarization cannot be ignored because it significantly decreases the relaxation time of the Stern layer of calcite.

\section{CONCLUSIONS}

A mechanistic complex conductivity model was developed to interpret complex conductivity measurements of calcite precipitation on glass beads. The complex conductivity model was combined with a basic Stern model (BSM) of the calcite/water interface and considers the electrochemical polarization of the Stern and diffuse layers surrounding calcite particles. In our conductivity model, the Stern layer is assumed to control the polarization response of the porous medium. However, the effects of the diffuse layer on the polarization of the Stern layer are also considered. The polarized diffuse layer is assumed to decrease the relaxation time of the polarized Stern layer. Our complex conductivity model depends on the surface charge density of the Stern layer and on the electrical potential at the onset of the diffuse layer, which were computed by our BSM and kept constant during the simulation of the calcite precipitation experiment. The particle size distribution and cementation exponent describing the shape of calcite particles were inverted from imaginary conductivity measurements.

Our complex conductivity model reproduces very well the complex conductivity measurements and gives the evolution of the particle size distribution and shape during the calcite precipitation experiment. These geometrical parameters are of primary importance to monitor the transport properties such as the permeability of sandstones containing saline solutions favouring calcite precipitation. Adjusted particle size distribution and cementation exponent were in agreement with the microscopy observations of the evolution of the pore structure and connectivity during calcite 
precipitation. The kinetics of calcite precipitation on glass beads are described by considering two different stages of calcite precipitation, one before the pores clogging where modelled particle size increases with time due to the growth of discrete calcite crystals, and another during the start of the pores clogging where only the smaller particles may influence the polarization response. During the first stage of calcite precipitation, the cementation exponent of calcite particles increases with time because of the flattening of precipitating calcite particles. Individual calcite crystals aggregate into larger but flatter calcite grains because precipitating calcite crystals grow laterally and come in contact with each other. During the second stage of calcite precipitation, the cementation exponent of calcite particles decreases with time because only dispersed and smaller calcite crystals may polarize. Aggregates of calcite particles lying over multiple glass beads may not polarize sufficiently in the investigated frequency range $[0.1-10000 \mathrm{~Hz}]$ at this stage. Our combined surface complexation and complex conductivity models also show that the Stern layer of calcite may control the polarization response of glass beads covered by calcite particles. Our models show in addition that the polarization of the diffuse layer decreases significantly the relaxation time of the polarized Stern layer because of the high surface charge density of the Stern layer and of the low differential capacitance of the diffuse layer.

In the future, spectral induced polarization measurements will be performed on calcite considering the effects of the impedances of the potential electrodes on the measured voltage. Additional characterization measurements will also be performed to check the electrochemical parameters such as the zeta potential and the geometrical parameters of the complex conductivity model such as the particle size distribution and the particles shape.

\section{ACKNOWLEDGEMENTS}

This work was supported by the BRGM-Carnot Institute, the Horizon 2020 CEBAMA (Contract Number: 662147), and ANR (Agence Nationale de la Recherche) CGS $\mu$ Lab (ANR-12-SEED0002) projects. We are indebted to Dr Mohamed Azaroual and Francis Claret for their support through the BRGM-Carnot Institute. Dr Shuai Li post-doctoral grant was supported by the BRGM-Carnot Institute. We thank warmly Dr Nicolas Devau for fruitful discussions. We also thank very much the Editor, Prof Georg Dresen, and Prof Lee Slater and the anonymous reviewer for their thorough review and constructive comments of our manuscript.

\section{REFERENCES}

Bernard, O., Kunz, W., Turq, P. \& Blum, L., 1992. Self-diffusion in electrolyte-solutions using the mean spherical approximation, J. Phys. Chem.-Us., 96, 398-403.

Bikerman, J.J., 1940. Electrokinetic equations and surface conductance. A survey of the diffuse double layer theory of colloidal solutions, Trans. Faraday Soc., 35, 154-160.

Bruggeman, D.A.G., 1935. Berechnung verschiedener physikalischer Konstanten von heterogenen Substanzen, Annalen der Physik, 416, 636-664.

Bucker, M. \& Hordt, A., 2013. Analytical modelling of membrane polarization with explicit parametrization of pore radii and the electrical double layer, Geophys. J. Int., 194, 804-813.

Chapman, D.L., 1913. A contribution to the theory of electrocapillarity, Philos. Mag. Serie 6, 25, 475-481.

Chelidze, T.L. \& Gueguen, Y., 1999. Electrical spectroscopy of porous rocks: a review-I. Theoretical models, Geophys. J. Int., 137, 1-15.
Cheng, L.W., Fenter, P., Sturchio, N.C., Zhong, Z. \& Bedzyk, M.J., 1999. $\mathrm{X}$-ray standing wave study of arsenite incorporation at the calcite surface, Geochim. Cosmochim. Acta, 63, 3153-3157.

Cole, K.S. \& Cole, R.H., 1941. Dispersion and absorption in dielectrics. I. Alternating current characteristics, J. Chem. Phys., 9, 341-351.

Collet, L.S., 1990. History of induced polarization method, in Induced Polarization: Applications and Case Histories, pp. 5-22, eds Fink, J.B., McAlister, E.O., Sternberg, B.K., Widuwilt, W.G. \& Ward, S.H., Society of Exploration Geophysics.

Davis, J.A., James, R.O. \& Leckie, J.O., 1978. Surface ionization and complexation at oxide-water interface. I. Computation of electrical double-layer properties in simple electrolytes, J. Colloid Interf. Sci., 480-499.

DeJong, J.T., Fritzges, M.B. \& Nusslein, K., 2006. Microbially induced cementation to control sand response to undrained shear, J. Geotech. Geoenviron., 132, 1381-1392.

DeJong, J.T., Mortensen, B.M., Martinez, B.C. \& Nelson, D.C., 2010. Biomediated soil improvement, Ecol. Eng., 36, 197-210.

Delgado, A.V., Arroyo, F.J., Gonzalez-Caballero, F., Shilov, V.N. \& Borkovskaya, Y.B., 1998. The effect of the concentration of dispersed particles on the mechanisms of low-frequency dielectric dispersion (LFDD) in colloidal suspensions, Colloid Surf. A, 140, 139-149.

Delima, O.A.L. \& Sharma, M.M., 1992. A generalized Maxwell-Wagner theory for membrane polarization in shaly sands, Geophysics, 57, 431440.

Dukhin, S.S. \& Shilov, V.N., 1974. Dielectric Phenomena and the Double Layer in Disperse Systems and Polyelectrolytes, Wiley.

Eriksson, R., Merta, J. \& Rosenholm, J.B., 2007. The calcite/water interface. I. Surface charge in indifferent electrolyte media and the influence of lowmolecular-weight polyelectrolyte, J. Colloid Interf. Sci., 313, 184-193.

Florsch, N., Revil, A. \& Camerlynck, C., 2014. Inversion of generalized relaxation time distributions with optimized damping parameter, J. appl. Geophys., 109, 119-132.

Fujita, Y., Redden, G.D., Ingram, J.C., Cortez, M.M., Ferris, F.G. \& Smith, R.W., 2004. Strontium incorporation into calcite generated by bacterial ureolysis, Geochim. Cosmochim. Acta, 68, 3261-3270.

Gouy, G., 1910. Sur la constitution de la charge électrique a surface d'un électrolyte, Annales de Physique (Paris) Série 4, 9, 457-468.

Grosse, C. \& Foster, K.R., 1987. Influence of bulk diffusion on the counterion polarization in a condensed counterion model, J. Phys. Chem.-Us., 91, 6415-6417.

Guichet, X., Jouniaux, L. \& Catel, N., 2006. Modification of streaming potential by precipitation of calcite in a sand-water system: laboratory measurements in the $\mathrm{pH}$ range from 4 to 12 , Geophys. J. Int., 166, 445460.

Hanai, T., 1968. 5 Electrical properties of emulsions, in Emulsions Science, pp. 354-477, ed. Sherman, P., Academic Press.

Heberling, F., Trainor, T.P., Lutzenkirchen, J., Eng, P., Denecke, M.A. \& Bosbach, D., 2011. Structure and reactivity of the calcite-water interface, J. Colloid Interf. Sci., 354, 843-857.

Heberling, F. et al., 2014. Reactivity of the calcite-water-interface, from molecular scale processes to geochemical engineering, Appl. Geochem., 45, 158-190.

Heuser, M., Spagnoli, G., Leroy, P., Klitzsch, N. \& Stanjek, H., 2012. Electro-osmotic flow in clays and its potential for reducing clogging in mechanical tunnel driving, B. Eng. Geol. Environ., 71, 721-733.

Hiemstra, T. \& Van Riemsdijk, W.H., 2006. On the relationship between charge distribution, surface hydration, and the structure of the interface of metal hydroxides, J. Colloid Interf. Sci., 301, 1-18.

Hiemstra, T. \& VanRiemsdijk, W.H., 1996. A surface structural approach to ion adsorption: The charge distribution (CD) model, J. Colloid Interf. Sci., 179, 488-508.

Huisman, J.A., Zimmermann, E., Esser, O., Haegel, F.-H., Treichel, A. \& Vereecken, H., 2015. Evaluation of a novel correction procedure to remove electrode impedance effects from broadband SIP measurements, J. appl. Geophys, 135, 466-473.

Hunter, R.J., 1981. Zeta Potential in Colloid Science: Principles and Applications, Academic Press. 
Jeen, S.W., Jambor, J.L., Blowes, D.W. \& Gillham, R.W., 2007. Precipitates on granular iron in solutions containing calcium carbonate with trichloroethene and hexavalent chromium, Environ. Sci. Technol., 41, 1989-1994.

Jougnot, D., Ghorbani, A., Revil, A., Leroy, P. \& Cosenza, P., 2010. Spectral induced polarization of partially saturated clay-rocks: a mechanistic approach, Geophys. J. Int., 180, 210-224.

Jougnot, D., Revil, A. \& Leroy, P., 2009. Diffusion of ionic tracers in the Callovo-Oxfordian clay-rock using the Donnan equilibrium model and the formation factor, Geochim. Et Cosmochim. Acta, 73, 2712-2726.

Kemna, A. et al., 2012. An overview of the spectral induced polarization method for near-surface applications, Near Surf. Geophys., 10, 453-468.

Kile, D.E., Eberl, D.D., Hoch, A.R. \& Reddy, M.M., 2000. An assessment of calcite crystal growth mechanisms based on crystal size distributions, Geochim. Cosmochim. Acta, 64, 2937-2950.

Leroy, P., Devau, N., Revil, A. \& Bizi, M., 2013. Influence of surface conductivity on the apparent zeta potential of amorphous silica nanoparticles, J. Colloid Interf. Sci., 410, 81-93.

Leroy, P., Jougnot, D., Revil, A., Lassin, A. \& Azaroual, M., 2012. A double layer model of the gas bubble/water interface, J. Colloid Interf. Sci., 388, 243-256.

Leroy, P. \& Revil, A., 2004. A triple-layer model of the surface electrochemical properties of clay minerals, J. Colloid Interf. Sci., 270, 371-380.

Leroy, P. \& Revil, A., 2009. A mechanistic model for the spectral induced polarization of clay materials, J. geophys. Res.-Solid Earth, 114, 1-21.

Leroy, P., Revil, A. \& Coelho, D., 2006. Diffusion of ionic species in bentonite, J. Colloid Interf. Sci., 296, 248-255.

Leroy, P., Revil, A., Kemna, A., Cosenza, P. \& Ghorbani, A., 2008. Complex conductivity of water-saturated packs of glass beads, J. Colloid Interf. Sci., 321, 103-117.

Leroy, P., Tournassat, C., Bernard, O., Devau, N. \& Azaroual, M., 2015. The electrophoretic mobility of montmorillonite. Zeta potential and surface conductivity effects, J. Colloid Interf. Sci., 451, 21-39.

Leroy, P., Tournassat, C. \& Bizi, M., 2011. Influence of surface conductivity on the apparent zeta potential of $\mathrm{TiO} 2$ nanoparticles, J. Colloid Interf. Sci., 356, 442-453.

Lesmes, D.P. \& Morgan, F.D., 2001. Dielectric spectroscopy of sedimentary rocks, J. geophys. Res.-Solid Earth, 106, 13 329-13 346.

Li, L., Gawande, N., Kowalsky, M.B., Steefel, C.I. \& Hubbard, S.S., 2011. Physicochemical heterogeneity controls on uranium bioreduction rates at the field scale, Environ. Sci. Technol., 45, 9959-9966.

Li, L., Steefel, C.I., Williams, K.H., Wilkins, M.J. \& Hubbard, S.S., 2009. Mineral transformation and biomass accumulation associated with uranium bioremediation at Rifle, Colorado, Environ. Sci. Technol., 43, 54295435.

Li, S., Leroy, P., Heberling, F., Devau, N., Jougnot, D. \& Chiaberge, C., 2016. Influence of surface conductivity on the apparent zeta potential of calcite, J. Colloid Interf. Sci., 468, 262-275.

Lide, D.R., 1990. CRC Handbook of Chemistry and Physics, CRC Press.

Luo, Y. \& Zhang, G., 1998. Theory and Application of Spectral Induced Polarization, Vol. 8, Society of Exploration Geophysicists.

Lyklema, J., 1991. Fundamentals of Interface and Colloid Science. Volume I: Fundamentals, Academic Press.

Lyklema, J., 1995. Fundamentals of Interface and Colloid Science. Volume II: Solid-Liquid Interfaces, Academic Press.

Lyklema, J., Dukhin, S.S. \& Shilov, V.N., 1983. The Relaxation of the double-layer around colloidal particles and the low-frequency dielectricdispersion .1. Theoretical considerations, J. Electroanal Chem., 143, 121.

Lyklema, J. \& Minor, M., 1998. On surface conduction and its role in electrokinetics, Colloids Surf A-Physicochem. Eng. Aspects, 140, 3341.

Lyklema, J., Rovillard, S. \& De Coninck, J., 1998. Electrokinetics: the properties of the stagnant layer unraveled, Langmuir, 14, 5659-5663.

Mendelson, K.S. \& Cohen, M.H., 1982. The effect of grain anisotropy on the electrical-properties of sedimentary-rocks, Geophysics, 47, 257-263.

Mitchell, A.C. \& Ferris, F.G., 2005. The coprecipitation of Sr into calcite precipitates induced by bacterial ureolysis in artificial groundwater: temperature and kinetic dependence, Geochim. Cosmochim. Acta, 69, 4199-4210.

Niu, Q., Revil, A. \& Saidian, M., 2016. Salinity dependence of the complex surface conductivity of the Portland sandstone, Geophysics, 81, D125D140.

O'Konski, C.T., 1960. Electric properties of macromolecules. V. Theory of ionic polarization in polyelectrolytes, J. Phys. Chem., 64, 605-619.

Okay, G., Leroy, P., Ghorbani, A., Cosenza, P., Camerlynck, C., Cabrera, J., Florsch, N. \& Revil, A., 2014. Spectral induced polarization of clay-sand mixtures: experiments and modeling, Geophysics, 79, E353-E375.

Olhoeft, G.R., 1981. Electrical properties of rocks, in Physical Properties of Rocks and Minerals, pp. 257-339, eds Touloukian, Y.S., Judd, W.R. \& Roy, R.F., McGraw-Hill.

Pacios, M., Hosseini, P., Fan, Y., He, Z., Krause, O., Hutchison, J., Warner, J.H. \& Bhaskaran, H., 2016. Direct manufacturing of ultrathin graphite on three-dimensional nanoscale features, Scient. Rep., 6, 22700.

Parkhurst, D.L. \& Appelo, C.A.J., 2013. Description of input and examples for PHREEQC version 3-A computer program for speciation, batchreaction, one-dimensional transport, and inverse geochemical calculations: U.S. Geological Survey Techniques and Methods, book 6, chap. A43, 497 p., Available at: https://pubs.usgs.gov/tm/06/a43/.

Pelton, W.H., Ward, S.H., Hallof, P.G., Sill, W.R. \& Nelson, P.H., 1978. Mineral discrimination and removal of inductive coupling with multifrequency-Ip, Geophysics, 43, 588-609.

Press, W.H., Flannery, B.P., Teukolsky, S.A. \& Vetterling, W.T., 1989. Numerical Recipes in Pascal: The Art of Scientific Computing, Cambridge Univ. Press.

Pruess, K., Xu, T.F., Apps, J. \& Garcia, J., 2003. Numerical modeling of aquifer disposal of $\mathrm{CO}_{2}, S P E J ., 8,49-60$.

Revil, A., 2000. Thermal conductivity of unconsolidated sediments with geophysical applications, J. geophys. Res.-Solid Earth, 105, 16 74916768 .

Revil, A., 2012. Spectral induced polarization of shaly sands: influence of the electrical double layer, Water Resour. Res., 48, 1-23.

Revil, A. \& Cathles, L.M., 1999. Permeability of shaly sands, Water Resour. Res., 35, 651-662.

Revil, A., Cathles, L.M., Losh, S. \& Nunn, J.A., 1998. Electrical conductivity in shaly sands with geophysical applications, J. geophys. Res.-Solid Earth, 103, 23 925-23936.

Revil, A. \& Florsch, N., 2010. Determination of permeability from spectral induced polarization in granular media, Geophys. J. Int., 181, 1480-1498.

Revil, A. \& Glover, P.W.J., 1997. Theory of ionic-surface electrical conduction in porous media, Phys. Rev. B., 55, 1757-1773.

Revil, A. \& Leroy, P., 2004. Constitutive equations for ionic transport in porous shales, J. geophys. Res.-Solid Earth, 109, 1-19.

Revil, A. \& Linde, N., 2006. Chemico-electromechanical coupling in microporous media, J. Colloid Interf. Sci., 302, 682-694.

Revil, A., Leroy, P. \& Titov, K., 2005. Characterization of transport properties of argillaceous sediments: application to the CallovoOxfordian argillite, J. geophys. Res.-Solid Earth, 110, B06202, doi:10.1029/2004JB003442.

Ricci, M., Spijker, P., Stellacci, F., Molinari, J.F. \& Voitchovsky, K., 2013. Direct visualization of single ions in the stern layer of calcite, Langmuir, 29, 2207-2216.

Scheibe, T.D., Fang, Y.L., Murray, C.J., Roden, E.E., Chen, J.S., Chien, Y.J., Brooks, S.C. \& Hubbard, S.S., 2006. Transport and biogeochemical reaction of metals in a physically and chemically heterogeneous aquifer, Geosphere, 2, 220-235.

Schurr, J.M., 1964. On the theory of the dielectric dispersion of spherical colloidal particles in electrolyte solution, J. Phys. Chem., 68, 2407-2413.

Schwarz, G., 1962. A theory of the low-frequency dielectric dispersion of colloidal particles in electrolyte solution, J. Phys. Chem., 66, 2636-2642.

Sen, P.N., Scala, C. \& Cohen, M.H., 1981. A self-similar model for sedimentary rocks with application to the dielectric constant of fused glass beads, Geophysics, 46, 781-795.

Stern, O., 1924. Zur Theorie der Electrolytischen Doppelschicht, Zeitschrift für Elektrochemie, 30, 508-516. 
Stipp, S.L.S., 1999. Toward a conceptual model of the calcite surface: hydration, hydrolysis, and surface potential, Geochim. Cosmochim. Acta, 63, 3121-3131.

Sturchio, N.C. et al., 1997. Lead adsorption at the calcite-water interface: synchrotron X-ray standing wave and X-ray reflectivity studies, Geochim. Cosmochim. Acta, 61, 251-263.

Sverjensky, D., 2005. Prediction of surface charge on oxides in salt solutions: revisions for 1: 1 (M + L-) electrolytes, Geochim. Cosmochim. Acta, 69, $225-257$.

Sverjensky, D.A., 2001. Interpretation and prediction of triple-layer model capacitances and the structure of the oxide-electrolyte-water interface, Geochim. Cosmochim. Acta, 65, 3643-3655.

Vancappellen, P., Charlet, L., Stumm, W. \& Wersin, P., 1993. A surface complexation model of the carbonate mineral-aqueous solution interface, Geochim. Cosmochim. Acta, 57, 3505-3518.

Vaudelet, P., Revil, A., Schmutz, M., Franceschi, M. \& Begassat, P., 2011. Induced polarization signatures of cations exhibiting differential sorption behaviors in saturated sands, Water Resour. Res., 47, 1-21.

Vinegar, H.J. \& Waxman, M.H., 1984. Induced polarization of shaly sands, Geophysics, 49, 1267-1287.

Weller, A. \& Borner, F.D., 1996. Measurements of spectral induced polarization for environmental purposes, Environ. Geol., 27, 329-334.

Weller, A., Slater, L. \& Nordsiek, S., 2013. On the relationship between induced polarization and surface conductivity: implications for petrophysical interpretation of electrical measurements, Geophysics, 78, D315D325.

Whiffin, V.S., van Paassen, L.A. \& Harkes, M.P., 2007. Microbial carbonate precipitation as a soil improvement technique, Geomicrobiol. J., 24, 417423.

Wilkin, R.T., Puls, R.W. \& Sewell, G.W., 2003. Long-term performance of permeable reactive barriers using zero-valent iron: geochemical and microbiological effects, Ground Water, 41, 493-503.

Wolthers, M., Charlet, L. \& Van Cappellen, P., 2008. The surface chemistry of divalent metal carbonate minerals; a critical assessment of surface charge and potential data using the charge distribution multi-site ion complexation model, Am. J. Sci., 308, 905-941.

Wolthers, M., Nehrke, G., Gustafsson, J.P. \& Van Cappellen, P., 2012. Calcite growth kinetics: modeling the effect of solution stoichiometry, Geochim. Cosmochim. Acta, 77, 121-134.

Wu, Y.X., Hubbard, S., Williams, K.H. \& Ajo-Franklin, J., 2010. On the complex conductivity signatures of calcite precipitation, J. geophys. Res.Biogeo, 115, 1-10.

Wu, Y.X. et al., 2011. Geophysical monitoring and reactive transport modeling of ureolytically-driven calcium carbonate precipitation, Geochem. T., 12, 1-20.

Xu, T.F., Apps, J.A. \& Pruess, K., 2003. Reactive geochemical transport simulation to study mineral trapping for $\mathrm{CO}_{2}$ disposal in deep arenaceous formations, J. geophys. Res.-Solid Earth, 108, 1-13.

Ye, K., Wang, X., Cao, L., Li, S., Li, Z., Yu, L. \& Ding, J., 2015. Matrix stiffness and nanoscale spatial organization of cell-adhesive ligands direct stem cell fate, Nano Lett., 15, 4720-4729.

Zhang, C., Slater, L., Redden, G., Fujita, Y., Johnson, T. \& Fox, D., 2012. Spectral induced polarization signatures of hydroxide adsorption and mineral precipitation in porous media, Environ. Sci. Technol., 46, 4357-4364.

\section{APPENDIX A}

In this appendix, we show a method to obtain an analytical solution of the Schwarz (1962) theory to describe Stern layer polarization when different types of monovalent and multivalent counter-ions are adsorbed at the Stern layer, following the calculations of Lyklema et al. (1983).

A spherical particle of radius $a$ covered by a thin layer of bound counter-ions and immersed in an aqueous electrolyte is considered. The particle has a complex electrical conductivity $\sigma_{s}^{*}$ and dielectric permittivity $\varepsilon_{s}{ }^{*}$ and is surrounded by an aqueous electrolyte of complex electrical conductivity $\sigma_{w}^{*}$ and dielectric permittivity $\varepsilon_{w}{ }^{*}$.
At thermodynamic equilibrium (absence of ions fluxes), ions are assumed homogeneously distributed in the thin layer of bound counter-ions, which has an initial surface charge density $Q_{b}^{0}$ (in $\mathrm{C} \mathrm{m}^{-2}$ ) (Davis et al. 1978). According to the triple layer model (TLM) of Davis et al. (1978), the surface charge density of the Stern layer can be computed as a function of the surface site densities of adsorbed counter-ions and is described by:

$Q_{b}^{0}=\sum_{i=1}^{P} q_{i} \Gamma_{i}^{b 0}$,

where $P$ is the number of different counter-ions in the Stern layer, $q_{i}= \pm e z_{i}$ is the ion charge (' + ' stands for cations and '-' stands for anions), $e$ is the elementary charge (of value $\sim 1.602 \times 10^{-19} \mathrm{C}$ ), $z_{i}$ is the ions valence, and $\Gamma_{i}^{b 0}$ is the surface site density of bound counterions $i$ (for instance $\mathrm{Na}^{+}$or $\mathrm{Ca}^{2+}$ ) at thermodynamic equilibrium (in sites $\left.\mathrm{m}^{-2}\right)$.

The medium is now submitted to an external alternating electric field of angular frequency $\omega$ (in $\mathrm{rad} \mathrm{s}^{-1}$ ) whose magnitude $E$ (in $\mathrm{V} \mathrm{m}^{-1}$ ) is given by:

$E=E_{0} \mathrm{e}^{i \omega t}$,

where $t$ is the time (in s).

When the alternating electric field is applied to the particle, the counter-ions distribution is not homogeneous anymore, and the surface charge density and surface site density of adsorbed counter-ions become $Q_{b}$ and $\Gamma_{i}^{b}$, respectively. These two parameters are linked to each other by:

$Q_{b}=\sum_{i=1}^{P} q_{i} \Gamma_{i}^{b}$.

The electric field along the surface is of the same order of magnitude as the external electric field or even smaller, and hence the surface charge density and counter-ions surface site density deviate only little from their random distribution, that is:

$Q_{b}-Q_{b}^{0}=\bar{Q}_{b} \ll Q_{b}^{0}$,

$\Gamma_{i}^{b}-\Gamma_{i}^{b 0}=\bar{\Gamma}_{i}^{b} \ll \Gamma_{i}^{b 0}$.

Furthermore, according to eqs (A2), (A4) and (A5), we obtain:

$\frac{\partial Q_{b}}{\partial t}=\frac{\partial \bar{Q}_{b}}{\partial t}=i \omega \bar{Q}_{b}$,

$\Gamma_{i}^{b} \cong \Gamma_{i}^{b 0}$.

According to Schwarz (1962), under the applied alternating electric field, counter-ions in the Stern layer first electromigrate along the particle surface and then diffuse back to restore their initial homogeneous distribution. These ions surface fluxes in the Stern layer can be described by the Nernst-Planck equation, which is written as (Revil \& Leroy 2004; Revil \& Linde 2006):

$\mathbf{j}_{i}=\mathbf{j}_{i}^{e}+\mathbf{j}_{i}^{d}+\mathbf{j}_{w}=-q_{i} u_{i}^{b} \Gamma_{i}^{b} \nabla \varphi_{b}-u_{i}^{b} k_{B} T \nabla \Gamma_{i}^{b}+\Gamma_{i}^{b} \mathbf{v}_{\mathbf{w}}$,

where $\mathbf{j}_{i}$ is the total surface flux, $\mathbf{j}_{i}^{e}$ is the surface electromigration flux, and $\mathbf{j}_{i}^{d}$ is the surface diffusion flux of ion $i$ (in mol $\mathrm{m}^{-2} \mathrm{~s}^{-1}$ ), $\mathbf{j}_{w}$ is the surface water flux (electro-osmosis), $u_{i}^{b}$ is the tangential velocity of ion $i$ per unit force (in $\mathrm{m}^{2} \mathrm{~s}^{-1} \mathrm{~V}^{-1} \mathrm{C}^{-1}, u_{i}^{b}=\beta_{i}^{b} /\left|q_{i}\right|$ ), $\varphi_{b}$ is the electrical potential at the Stern plane (in V), $k_{B}$ is the Boltzmann constant (of value $\sim 1.381 \times 10^{-23} \mathrm{~J} \mathrm{~K}^{-1}$ ), and $\mathbf{v}_{\mathbf{w}}$ is the local velocity of the pore water (in $\mathrm{m} \mathrm{s}^{-1}$ ).

No water flow is considered in the Stern layer because of the high viscosity of this layer (Lyklema et al. 1998). This implies 
that $\mathbf{v}_{\mathbf{w}}=0 \mathrm{~m} \mathrm{~s}^{-1}$ in the Stern layer. This assumption considerably simplifies the calculation of the ions surface fluxes in the Stern layer because only the Nernst-Planck equation must be solved to describe the ions surface fluxes, that is it is not necessary to solve simultaneously the Nernst-Planck and Navier-Stokes equations (for the water flow) to describe the ions and water surface fluxes.

We consider now a spherical coordinate system centred on the particle, that is with $r=0$ at the centre of the particle and with $\theta=0$ in the direction of $\boldsymbol{E}$. In absence of water flow (electro-osmosis is neglected), and in spherical coordinates, eq. (A8) then becomes:

$\mathbf{j}_{i}=-\frac{q_{i} u_{i}^{b} \Gamma_{i}^{b}}{a} \frac{\partial \varphi_{b}}{\partial \theta}-\frac{u_{i}^{b} k_{B} T}{a} \frac{\partial \Gamma_{i}^{b}}{\partial \theta}$.

The total current density in the bound layer $\mathbf{J}_{s}\left(\right.$ in $\mathrm{A} \mathrm{m}^{-2}$ ) is written as:

$\mathbf{J}_{s}=\sum_{i=1}^{P} q_{i} \mathbf{j}_{i}$

By combining eqs (A9) and (A10), the total current density resulting from electrochemical polarization of the Stern layer is written as:

$\mathbf{J}_{s}=-\frac{1}{a}\left(\sum_{i=1}^{P} q_{i} \Gamma_{i}^{b} q_{i} u_{i}^{b} \frac{\partial \varphi_{b}}{\partial \theta}+\sum_{i=1}^{P} q_{i} u_{i}^{b} k_{B} T \frac{\partial \Gamma_{i}^{b}}{\partial \theta}\right)$,

$\mathbf{J}_{s}=-\frac{1}{a}\left(\sum_{i=1}^{P} q_{i} \Gamma_{i}^{b} \frac{q_{i} D_{i}^{b}}{k_{B} T} \frac{\partial \varphi_{b}}{\partial \theta}+\sum_{i=1}^{P} q_{i} D_{i}^{b} \frac{\partial \Gamma_{i}^{b}}{\partial \theta}\right)$,

where $D_{i}^{b}=u_{i}^{b} k_{B} T$ is the tangential diffusion coefficient of the counter-ions in the Stern layer (in $\mathrm{m}^{2} \mathrm{~s}^{-1}$ ).

We make now the assumption that all the different counter-ions in the Stern layer have the same tangential diffusion coefficient $D_{b}$. Furthermore, the surface site densities of counter-ions in the Stern layer only deviate little from their initial distribution, that is $\Gamma_{i}^{b} \cong \Gamma_{i}^{b 0}$. Under these two assumptions, eq. (A12) becomes:

$\mathbf{J}_{s}=-\frac{D_{b}}{a}\left(\sum_{i=1}^{P} q_{i} \Gamma_{i}^{b 0} \frac{q_{i}}{k_{B} T} \frac{\partial \varphi_{b}}{\partial \theta}+\sum_{i=1}^{P} q_{i} \frac{\partial \Gamma_{i}^{b}}{\partial \theta}\right)$.

Seeing eqs (A1) and (A13), it becomes now obvious to relate the surface current density $\mathbf{J}_{s}$ to the surface charge density of the Stern layer at thermodynamic equilibrium $Q_{b}^{0}$ by making a third approximation regarding the electromigration flux of counter-ions (first term of eq. A13). We assume in eq. (A13) that $q_{i}=q$ with $q$ the averaged electrical charge of the counter-ions in the Stern layer. Following this assumption and using eqs (A1) and (A4), eq. (A13) becomes:

$\mathbf{J}_{s}=-\frac{D_{b}}{a}\left(\frac{q Q_{b}^{0}}{k_{B} T} \frac{\partial \varphi_{b}}{\partial \theta}+\frac{\partial \bar{Q}_{b}}{\partial \theta}\right)$,

which is very close to the eq. (6) of Lyklema et al. (1983).

The Schwarz (1962) charge conservation condition contains no normal flow and is written (Lyklema et al. 1983):

$\nabla \cdot \mathbf{J}_{s}=-\frac{\partial Q_{b}}{\partial t}$.

By combining eqs (A6), (A14) and (A15), we obtain in the spherical coordinate system (Lyklema et al. 1983):

$i \omega \bar{Q}_{b}=\frac{D_{b}}{a^{2}} \frac{1}{\sin \theta} \frac{\partial}{\partial \theta}\left[\sin \theta\left(\frac{q Q_{b}^{0}}{k_{B} T} \frac{\partial \varphi_{b}}{\partial \theta}+\frac{\partial \bar{Q}_{b}}{\partial \theta}\right)\right]$,

which is similar to eq. (15) of Schwarz (1962) and eq. (19) of Lyklema et al. (1983). Therefore, eq. (A16) can be solved using the same boundary conditions than in Schwarz (1962) and its solution is (Schwarz 1962; Lyklema et al. 1983):

$\bar{Q}_{b}=-\frac{q Q_{b}^{0} / k_{B} T}{1+i \omega \tau_{b}} \varphi_{b}$,

$\tau_{b}=\frac{a^{2}}{2 D_{b}}$,

where $\tau_{b}$ is the relaxation time (in s) associated with the electrochemical polarization of the Stern layer. By replacing $\bar{Q}_{b}$ in eq. (A14) by its expression as a function of $\varphi_{b}$ in eq. (A17), the surface current density $\mathbf{J}_{s}$ can be expressed as a function of the gradient of the electrical potential at the Stern layer and hence surface electric field. It follows:

$\mathbf{J}_{s}=-\frac{D_{b} q Q_{b}^{0}}{a k_{B} T} \frac{i \omega \tau_{b}}{1+i \omega \tau_{b}} \frac{\partial \varphi_{b}}{\partial \theta} \mathbf{e}_{\theta}$,

$\mathbf{J}_{s}=\frac{D_{b} q Q_{b}^{0}}{k_{B} T} \frac{i \omega \tau_{b}}{1+i \omega \tau_{b}} \mathbf{E}_{s}$.

The surface current density can also be expressed as a function of the specific surface conductivity $\Sigma_{s}$ (in S) and surface electric field using

$\mathbf{J}_{s}=\Sigma_{s} \mathbf{E}_{s}$.

By combining eqs (A20) and (A21), the specific surface conductivity due to the polarization of the surface charge density of the Stern layer is given by:

$\Sigma_{s}=\Sigma_{s}^{b} \frac{i \omega \tau_{b}}{1+i \omega \tau_{b}}$

$\Sigma_{s}^{b}=\frac{D_{b} q Q_{b}^{0}}{k_{B} T}$.

In the main text of the manuscript, we choose to write only $Q_{b}$ for the surface charge density of the Stern layer at thermodynamic equilibrium to simplify the writing of the manuscript. Furthermore, the Nernst-Einstein equation can be used to simplify eq. (A23). The Nernst-Einstein equation is written as:

$D_{b}=\frac{k_{B} T}{|q|} \beta_{b}$,

where $\beta_{b}$ is the averaged ion mobility in the Stern layer (in $\mathrm{m}^{2} \mathrm{~s}^{-1} \mathrm{~V}^{-1}$ ). By combining eqs (A23) and (A24), we finally obtain this equation for the specific surface conductivity of the Stern layer:

$\Sigma_{s}^{b}= \pm \beta_{b} Q_{b}$,

where ' + ' stands for positive and '-' stands for negative surface charge density in the Stern layer, respectively ( $\Sigma_{s}^{b}$ is always positive).

Eq. (A25) shows that the specific surface conductivity of the Stern layer can be written as a function of the product of the averaged ion mobility in the Stern layer with the surface charge density of the Stern layer. Leroy et al. (2008) wrote that the specific surface conductivity of the Stern layer can be written as a function of the product of the ion mobility with the ion charge and the ion surface site density in the Stern layer (eq. 59 of Leroy et al. 2008). Therefore, our approach allows extending very easily the Stern layer polarization model of Leroy et al. (2008) to complex aqueous solutions using the assumption that all the counter-ions in the Stern layer have approximately the same tangential diffusion coefficient. 


\section{APPENDIX B}

Schwarz theory does not consider effects of the polarization of the diffuse layer on the polarization of the Stern layer (Lyklema et al. 1983; Ye et al. 2015; Niu et al. 2016). According to Lyklema et al. (1983), the Stern layer is almost completely screened by the diffuse layer and hence the polarization of the diffuse layer cannot be ignored.

Lyklema et al. (1983) considered the effects of the tangential oscillations of the diffuse layer on the polarization of bound ions in the Stern layer. For that purpose, they identified the electrical potential drop over the diffuse layer, $\varphi_{d}$, as the electrical potential drop between $r=a$ where $\varphi=\varphi_{b}$ and $r=a+\kappa^{-1}\left(\kappa^{-1}\right.$ is the Debye length, Hunter 1981) where $\varphi=\varphi_{e}$ (the subscript ' $e$ ' stands for external electrical potential), hence:

$\varphi_{d}=\varphi_{b}-\varphi_{e}$.

According to eq. (B1), the tangential electric field in the Stern layer is written as:

$\frac{\partial \varphi_{b}}{\partial \theta}=\frac{\partial \varphi_{e}}{\partial \theta}+\frac{\partial \varphi_{d}}{\partial \theta}$,

$\frac{\partial \varphi_{b}}{\partial \theta}=\frac{\partial \varphi_{e}}{\partial \theta}-\frac{1}{C_{d}} \frac{\partial Q_{d}}{\partial \theta}$,

where $C_{d}$ is the differential capacitance of the diffuse layer (in $\mathrm{C} \mathrm{m}^{-2} \mathrm{~V}^{-1}$ ) defined by (Hunter 1981):

$C_{d}=-\frac{\partial Q_{d}}{\partial \varphi_{d}}$.

Similarly to the surface charge density of the Stern layer, $Q_{b}$, the surface charge density of the diffuse layer, $Q_{d}$, can be written as a function of surface charge density of the diffuse layer at thermodynamic equilibrium, $Q_{d}^{0}$, and a small perturbation associated with diffuse layer polarization, $\bar{Q}_{d}$, hence:

$Q_{d}-Q_{d}^{0}=\bar{Q}_{d} \ll Q_{d}^{0}$,

$\frac{\partial Q_{d}}{\partial \theta}=\frac{\partial \bar{Q}_{d}}{\partial \theta}$.

The EDL as a whole is always approximately electroneutral at any $\theta$, hence:

$Q_{0}+Q_{b}+Q_{d}=0$,

where $Q_{0}$ is the surface charge density at the ' 0 -plane' located at the mineral surface (Hunter 1981).

By combining eqs (A4), (B5) and (B7) and considering electroneutrality condition for the EDL at thermodynamic equilibrium, we obtain:

$\bar{Q}_{b}=-\bar{Q}_{d}$,

which means that perturbation of the polarized diffuse layer exactly cancels perturbation of the polarized Stern layer.

By combining eqs (B3), (B6) and (B8), we obtain for the tangential electric field in the Stern layer:

$\frac{\partial \varphi_{b}}{\partial \theta}=\frac{\partial \varphi_{e}}{\partial \theta}+\frac{1}{C_{d}} \frac{\partial \bar{Q}_{b}}{\partial \theta}$.

By replacing $\partial \varphi_{b} / \partial \theta$ in eq. (A16) by its expression in eq. (B9), we obtain:

$$
\begin{aligned}
i \omega \bar{Q}_{b}= & \frac{D_{b}}{a^{2}} \frac{1}{\sin \theta} \frac{\partial}{\partial \theta} \\
& \times\left\{\sin \theta\left[\frac{q Q_{b}^{0}}{k_{B} T}\left(\frac{\partial \varphi_{e}}{\partial \theta}+\frac{1}{C_{d}} \frac{\partial \bar{Q}_{b}}{\partial \theta}\right)+\frac{\partial \bar{Q}_{b}}{\partial \theta}\right]\right\},
\end{aligned}
$$

$$
\begin{aligned}
i \omega \bar{Q}_{b}= & \frac{D_{b}}{a^{2}} \frac{1}{\sin \theta} \frac{\partial}{\partial \theta} \\
& \times\left\{\sin \theta\left[\frac{q Q_{b}^{0}}{k_{B} T} \frac{\partial \varphi_{e}}{\partial \theta}+\left(1+\frac{q Q_{b}^{0}}{k_{B} T C_{d}}\right) \frac{\partial \bar{Q}_{b}}{\partial \theta}\right]\right\} .
\end{aligned}
$$

By assuming that the differential capacitance of the diffuse layer, $C_{d}$, does not dependent on $\theta$, eq. (B11) becomes:

$i \omega \bar{Q}_{b}=\frac{D_{b} M}{a^{2}} \frac{1}{\sin \theta} \frac{\partial}{\partial \theta}\left\{\sin \theta\left[\frac{q Q_{b}^{0}}{k_{B} T} \frac{1}{M} \frac{\partial \varphi_{e}}{\partial \theta}+\frac{\partial \bar{Q}_{b}}{\partial \theta}\right]\right\}$,

$M=1+\frac{q Q_{b}^{0}}{k_{B} T C_{d}}$.

Eq. (B12) is similar to eq. (A16), except that $D_{b}$ and $\varphi_{b}$ in eq. (A16) are replaced by $D_{b} M$ and $\varphi_{e} / M$, respectively, in eq. (B12). Therefore, eq. (B12) can be solved using the same boundary conditions than in Schwarz (1962) and its solution is (Schwarz 1962; Lyklema et al. 1983):

$\bar{Q}_{b}=-\frac{q Q_{b}^{0} / k_{B} T}{1+i \omega \tau_{b}} \frac{\varphi_{e}}{M}$,

$\tau_{b}=\frac{a^{2}}{2 D_{b} M}$,

where $\tau_{b}$ is the relaxation time (in s) associated with the electrochemical polarization of the Stern layer.

By replacing in eq. (A14) $\partial \varphi_{b} / \partial \theta$ and $\partial \bar{Q}_{b} / \partial \theta$ by their expressions in eqs (B9) and (B14), respectively, the following equation is obtained for the surface current density:

$\mathbf{J}_{s}=-\frac{D_{b} q Q_{b}^{0}}{a k_{B} T}\left(\frac{i \omega \tau_{b}}{1+i \omega \tau_{b}} \frac{\partial \varphi_{e}}{\partial \theta}\right) \mathbf{e}_{\theta}$

$\mathbf{J}_{s}=\frac{D_{b} q Q_{b}^{0}}{k_{B} T}\left(\frac{i \omega \tau_{b}}{1+i \omega \tau_{b}}\right) \mathbf{E}_{s}$.

According to eqs (A21)-(A25) and (B17), the specific surface conductivity due to the polarization of the surface charge densities of the Stern and diffuse layers is given by:

$\Sigma_{s}=\Sigma_{s}^{b} \frac{i \omega \tau_{b}}{1+i \omega \tau_{b}}$,

$\Sigma_{s}^{b}= \pm \beta_{b} Q_{b}$.

Eqs (B15) and (B18) show that the polarized diffuse layer only decreases the relaxation time of bound counter-ions in the Stern layer because $M \geq 1$. The parameter $M$ can be calculated from the differential capacitance of the diffuse layer $C_{d}$, which can be computed from the derivative of the surface charge density of the diffuse layer $Q_{d}$ with respect to the electrical potential at the beginning of the diffuse layer $\varphi_{d}$ (eq. B4).

Assuming that the electrical potential in the diffuse layer only varies as a function of the distance of the ion from the beginning of the diffuse layer, the surface charge density of the diffuse layer is computed according to the volume charge density in the diffuse layer $\rho$ (in $\mathrm{C} \mathrm{m}^{-3}$ ) using (Hunter 1981):

$Q_{d}=\int_{x=0}^{x=\infty} \rho d x$

where $x$ is the distance of the ion from the beginning of the diffuse layer (in $\mathrm{m}$ ). According to the Poisson equation, the volume charge density in the diffuse layer is written as:

$\rho=-\varepsilon_{w} \frac{d^{2} \varphi}{d x^{2}}$ 
By combining eqs (B20) and (B21), and integrating the resulting equation with respect to $x$, we obtain:

$Q_{d}=\left.\varepsilon_{w} \frac{d \varphi}{d x}\right|_{x=0}$.

The Poisson-Boltzmann equation, which describes the electrical potential distribution at the solid/water or water/gas interface is (Hunter 1981; Lyklema 1995; Leroy et al. 2015):

$\frac{d^{2} \varphi(x)}{d x^{2}}=-\frac{1}{\varepsilon_{w}} \sum_{i=1}^{N} q_{i} 1000 N_{A} C_{i}^{w} \exp \left[-\frac{q_{i} \varphi(x)}{k_{B} T}\right]$,

where $N$ is the number of different types of counter-ions and coions in the diffuse layer and $N_{A}$ is the Avogadro number (of value $\sim 6.022 \times 10^{23} \mathrm{~mol}^{-1}$ ).

By multiplying both sides of eq. (B23) by $2 d \varphi / d x$ and integrating the resulting equation with respect to $x$, we obtain:

$$
\begin{aligned}
\int_{x=0}^{x=\infty} \frac{2 d \varphi}{d x} \frac{d^{2} \varphi}{d x^{2}} d x= & -\frac{2}{\varepsilon_{w}} \sum_{i=1}^{N} q_{i} 1000 N_{A} C_{i}^{w} \\
& \times \int_{\varphi=\varphi_{d}}^{\varphi=0} \exp \left(-\frac{q_{i} \varphi}{k_{B} T}\right) d \varphi \\
\int_{x=0}^{x=\infty} \frac{d}{d x}\left(\frac{d \varphi}{d x}\right)^{2} d x= & -\frac{2}{\varepsilon_{w}} \sum_{i=1}^{N} q_{i} 1000 N_{A} C_{i}^{w} \\
& \times \int_{\varphi=\varphi_{d}}^{\varphi=0} \exp \left(-\frac{q_{i} \varphi}{k_{B} T}\right) d \varphi
\end{aligned}
$$

$$
\left.\frac{d \varphi}{d x}\right|_{x=0}= \pm \sqrt{\frac{2 k_{b} T}{\varepsilon_{w}} \sum_{i=1}^{N} 1000 N_{A} C_{i}^{w}\left[\exp \left(-\frac{q_{i} \varphi_{d}}{k_{B} T}\right)-1\right]}
$$

The surface charge density of the diffuse layer is finally calculated as a function of the electrical potential at the beginning of the diffuse layer by combining eqs (B22) and (B26), hence:

$Q_{d}= \pm \sqrt{2 \varepsilon_{w} k_{b} T \sum_{i=1}^{N} 1000 N_{A} C_{i}^{w}\left[\exp \left(-\frac{q_{i} \varphi_{d}}{k_{B} T}\right)-1\right]}$

where ' + ' stands for negative electrical potentials $\varphi_{d}$ and '-' stands for positive electrical potentials $\varphi_{d}$.

By deriving $Q_{d}$ with respect to $\varphi_{d}$ in eq. (B27), we obtain an expression for the differential capacitance of the diffuse layer (eq. B4), hence:

$$
C_{d}=-\frac{\partial Q_{d}}{\partial \varphi_{d}}=\sqrt{\frac{\varepsilon_{w}}{2 k_{B} T}} \frac{\sum_{i=1}^{N} q_{i} 1000 N_{A} C_{i}^{w} \exp \left(-\frac{q_{i} \varphi_{d}}{k_{B} T}\right)}{\sqrt{\sum_{i=1}^{N} 1000 N_{A} C_{i}^{w}\left[\exp \left(-\frac{q_{i} \varphi_{d}}{k_{B} T}\right)-1\right]}},
$$

which can be used to compute the $M$ factor entering into eq. (B15) when different monovalent and multivalent ions are in the diffuse layer. 